\title{
GRB 071003: BROADBAND FOLLOW-UP OBSERVATIONS OF A VERY BRIGHT GAMMA-RAY BURST IN A GALACTIC HALO
}

\author{
D. A. Perley, ${ }^{1}$ W. Li, ${ }^{1}$ R. Chornock, ${ }^{1}$ J. X. Prochaska, ${ }^{2}$ N. R. Butler, ${ }^{1}$ P. Chandra,${ }^{3,4}$ L. K. Pollack, ${ }^{2}$ \\ J. S. Bloom, ${ }^{1,5}$ A. V. Filippenko, ${ }^{1}$ H. Swan, ${ }^{6}$ F. Yuan, ${ }^{6}$ C. Akerlof, ${ }^{6}$ M. W. Auger, ${ }^{7}$ S. B. Cenko, ${ }^{8}$ \\ H.-W. Chen, ${ }^{9}$ C. D. Fassnacht, ${ }^{7}$ D. Fox, ${ }^{8}$ D. Frail,${ }^{10}$ E. M. Johansson, ${ }^{11}$ T. McKay, ${ }^{6}$ \\ D. Le Mignant, ${ }^{2,11}$ M. Modjaz, ${ }^{1}$ W. Rujopakarn, ${ }^{12}$ R. Russel, ${ }^{13}$ M. A. Skinner, ${ }^{14}$ \\ G. H. Smith, ${ }^{2}$ I. Smith, ${ }^{15}$ M. A. van Dam, ${ }^{11}$ AND S. Yost ${ }^{16}$ \\ Received 2008 May 15; accepted 2008 July 15
}

\begin{abstract}
The optical afterglow of long-duration GRB 071003 is among the brightest yet to be detected from any GRB, with $R \approx 12$ mag in KAIT observations starting $42 \mathrm{~s}$ after the GRB trigger, including filtered detections during prompt emission. However, our high-S/N afterglow spectrum displays only extremely weak absorption lines at what we argue is the host redshift of $z=1.60435$, in contrast to the three other, much stronger Mg II absorption systems observed at lower redshifts. Together with Keck adaptive optics observations, which fail to reveal a host galaxy coincident with the burst position, our observations suggest a halo progenitor and offer a cautionary tale about the use of $\mathrm{Mg}$ II for GRB redshift determination. We present early- through late-time observations spanning the electromagnetic spectrum, constrain the connection between the prompt emission and early variations in the light curve (we observe no correlation), and discuss possible origins for an unusual, marked rebrightening that occurs a few hours after the burst: likely either a late-time refreshed shock or a wide-angle secondary jet. Analysis of the late-time afterglow is most consistent with a wind environment, suggesting a massive star progenitor. Together with GRB 070125, this may indicate that a small but significant portion of star formation in the early universe occurred far outside what we consider a normal galactic disk.
\end{abstract}

Subject headings: gamma rays: bursts

Online material: color figures, machine-readable table

\section{INTRODUCTION}

Concurrent observations of long-wavelength afterglow and ongoing gamma-ray burst (GRB) activity should, in principle, yield important constraints on the nature of the physical processes of the emission (e.g., Kobayashi 2000). However, as a GRB typically lasts less than $100 \mathrm{~s}$, it is challenging for large ground-based optical/ infrared follow-up facilities to react to a GRB alert quickly and take data during the prompt phase. Multicolor observations, which provide vital information on the emission mechanism, are even more difficult to obtain during the prompt phase because of the added overhead associated with changing filters. Nevertheless, due to the coordinated efforts of recent space missions (HETE-II,

\footnotetext{
1 Department of Astronomy, University of California, Berkeley, CA 947203411; dperley@astro.berkeley.edu,wli@astro.berkeley.edu.

2 Department of Astronomy and Astrophysics, University of California Observatories/Lick Observatory, University of California, Santa Cruz, CA 95064.

3 Department of Astronomy, University of Virginia, Charlottesville, VA 22904.

4 Jansky Fellow, National Radio Astronomy Observatory.

5 Sloan Research Fellow.

6 University of Michigan, Randall Laboratory of Physics, Ann Arbor, MI 48109-1040.

7 Department of Physics, University of California, Davis, CA 95616.

8 Division of Physics, Mathematics, and Astronomy, MS 105-24, California Institute of Technology, Pasadena, CA 91125.

9 Department of Astronomy and Astrophysics, University of Chicago, Chicago, IL 60637.

${ }^{10}$ National Radio Astronomy Observatory, Socorro, NM 87801.

11 W. M. Keck Observatory, Kamuela, HI 96743.

12 Steward Observatory, Tucson, AZ 85721.

13 The Aerospace Corporation, Los Angeles, CA 90009-29957.

14 The Boeing Company, Kihei, HI 96753.

15 Department of Physics and Astronomy, Rice University, Houston, TX 77251-1892.

${ }^{16}$ College of St. Benedict, St. Joseph, MN 56374.
}

Ricker et al. 2003; Swift, Gehrels et al. 2004) to detect GRBs and various ground-based optical follow-up programs, observations during the prompt phase of GRBs are no longer uncommon: the optical afterglows (OAs) of several dozen GRBs have been observed (e.g., Akerlof et al. 1999; Vestrand et al. 2006; Yost et al. 2007) during gamma-ray emission, and multicolor optical data have been obtained in a handful of cases (e.g., Blake et al. 2005; Nysewander et al. 2007).

Observations of GRBs in the past several years have also revealed a rich demography in OA behavior. Some OAs have monotonic power-law decays (e.g., Li et al. 2003b; Laursen \& Stanek 2003), while others have plateau (e.g., Rykoff et al. 2006) and rebrightening (e.g., Woźniak et al. 2006) phases. Even among GRBs with relatively simple behavior, however, short-timescale features not predicted in the basic shock models often appear in sufficiently well sampled data. Various modifications to the standard picture have been proposed to explain such observations, including the presence of a jet with single (e.g., Sari et al. 1999) or multiple (e.g., Berger et al. 2003) components, refreshed shocks (Zhang et al. 2006), central engine activity (Kocevski et al. 2007; Chincarini et al. 2007), gravitational microlensing (Garnavich et al. 2000), and density irregularity in the GRB environment (Holland et al. 2003). Observationally, constraints on the change in the afterglow color and the spectral energy distribution (SED) play an important role in limiting the viability of models for a particular GRB.

The question of the nature of the GRB itself is intimately tied to the question of its environment and origins. At intermediate to late times, spectroscopy of the afterglow (e.g., Prochaska et al. 2007; D'Elia et al. 2007) and deep imaging of the host environment (e.g., Bloom et al. 2002; Fruchter et al. 2006) can help establish the nature of the GRB's progenitor and environment, connecting what 
we learn about the burst itself to the larger question of its origins and place in the early universe.

In this paper we report on our photometric and spectroscopic observations of GRB 071003 with various telescopes from the prompt phase to late times. In $\S 2$ we describe the observations, and in $\S 3$ we present the reductions. The analysis of the light curves and the constraints on the changes in the colors and SEDs are given in $\S 4$. The conclusions, including the implications of the extremely unusual spectrum of this event, are discussed in $\S 5$. We assume $H_{0}=71 \mathrm{~km} \mathrm{~s}^{-1} \mathrm{Mpc}^{-1}, \Omega_{M}=0.3$, and $\Omega_{\Lambda}=0.7$ throughout the paper. These reports should be considered the final analysis of our group's data on GRB 071003, superseding any previously announced results (e.g., in the GCN Circulars).

\section{OBSERVATIONS}

\subsection{BAT/XRT Observations}

On 2007 October 3, 07:40:55 UT (defined as $t=0$ in this paper; UT dates are used throughout), a bright GRB triggered the Burst Alert Telescope (BAT) on board the Swift satellite (trigger 292934; Schady et al. 2007). The first GCN notice was distributed within $16 \mathrm{~s}$. Unfortunately, Swift was still returning to normal observations after its 2007 August gyro failure, but it did slew to the position after $22 \mathrm{ks}$ and began observations using the X-Ray Telescope (XRT).

We downloaded the Swift BAT and XRT data from the Swift Archive $^{17}$ and quicklook data site. ${ }^{18}$ The XRT and BAT spectra were fitted using ISIS. ${ }^{19}$

The XRT data were processed with version 0.11 .4 of the xrtpipeline reduction script from the HEAsoft $6.3 .1^{20}$ software release. We employ the latest (2007 December 4) XRT calibration files. Our reduction of XRT data from cleaned event lists output by xrtpipeline to science-ready light curves and spectra is described in detail by Butler \& Kocevski (2007a). We use the latest calibration files from the 2007 September 24 BAT database release. We establish the energy scale and mask weighting for the BAT event mode data by running the bateconvert and batmaskwtevt tasks. Spectra and light curves are extracted with the batbinevt task, and response matrices are produced by running batdrmgen. To produce the BAT spectra, we apply the systematic error corrections to the low-energy BAT spectral data as suggested by the BAT Digest Web site ${ }^{21}$ and fit the data in the $15-150 \mathrm{keV}$ band. The spectral normalizations are corrected for satellite slews using the batupdatephakw task.

The burst exhibits one dominant emission episode of duration $d t \approx 30 \mathrm{~s}$, followed by a minor pulse $\sim 150 \mathrm{~s}$ later of duration $\sim 20 \mathrm{~s}$. The total duration is $T_{90}=148 \pm 1 \mathrm{~s},{ }^{22}$ placing it clearly into the long GRB class. The primary pulse is resolved into multiple pulses. The gamma-ray light curve is shown in Figure 1, overplotted with early-time photometry from the Katzman Automatic Imaging Telescope (KAIT) and P60 (discussed in $\S \S 3.2$ and 3.5 , respectively).

The time-integrated BAT spectrum from $t=-10.3$ to $169 \mathrm{~s}$ is acceptably fitted $\left(\chi^{2} / \nu=47.64 / 55\right.$, where $\nu$ is the number of degrees of freedom) by a power-law model, with photon in$\operatorname{dex} \alpha=-1.3 \pm 0.1$ and energy fluence $S_{\gamma}=(1.7 \pm 0.1) \times$ $10^{-5} \mathrm{ergs} \mathrm{cm}^{-2}(15-350 \mathrm{keV})$. The main emission episode $(t=$ -1.4 to $22.8 \mathrm{~s})$ is harder $\left[\alpha=-1.08 \pm 0.03, S_{\gamma}=(1.51 \pm\right.$

\footnotetext{
17 See ftp:// legacy.gsfc.nasa.gov/swift/data.

18 See http://swift.gsfc.nasa.gov/cgi-bin/sdc/ql.

19 See http://space.mit.edu/CXC/ISIS.

20 See http://heasarc.gsfc.nasa.gov/docs/software/lheasoft/.

21 See http://swift.gsfc.nasa.gov/docs/swift/analysis/bat_digest.html.

22 All uncertainties quoted in this paper are $1 \sigma$, except where specified otherwise.
}

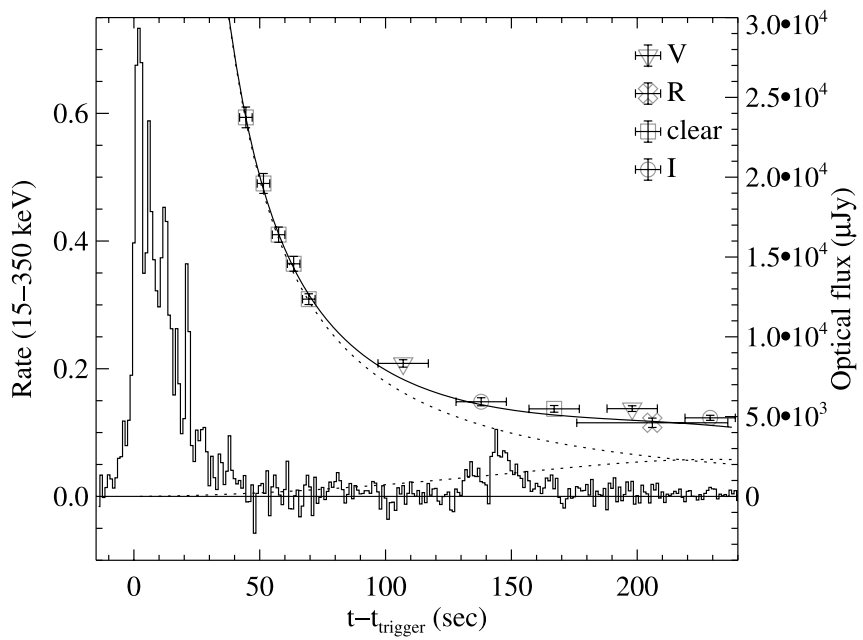

FIG. 1.- Light curve from the Swift BAT of GRB 071003, with optical photometry from KAIT and P60, and the optical light-curve model discussed in $\S 4.3$, overplotted. The GRB is dominated by a complicated, spiky emission episode in the first $30 \mathrm{~s}$, but a pulse is also observed much later, at $150 \mathrm{~s}$. Optical data points (all from KAIT, except one $R$-band measurement from the P60), by contrast, show a power-law decay at early times followed by a slow-rising "bump." Here the $V$ and $I$ filtered observations have been offset to match the $R$ and unfiltered points based on the relative colors at $2000 \mathrm{~s}$. [See the electronic edition of the Journal for a color version of this figure.]

$0.03) \times 10^{-5}$ ergs $\left.\mathrm{cm}^{-2}, \chi^{2} / \nu=56.71 / 55\right]$, while the final pulse $(t=131-169 \mathrm{~s})$ is softer $\left(\alpha=-1.8 \pm 0.2, S_{\gamma}=1.2_{-0.2}^{+0.1} \times\right.$ $\left.10^{-6} \mathrm{ergs} \mathrm{cm}^{-2}, \chi^{2} / \nu=41.15 / 55\right)$.

X-ray observations with the XRT began $6.2 \mathrm{hr}$ after the BAT trigger. The X-ray light curve measured until $t \approx 5 \times 10^{5} \mathrm{~s}$ is well fitted by a power-law time decay $t^{-1.68 \pm 0.03}$. The time-integrated spectrum is well fitted $\left(\chi^{2} / \nu=48.47 / 54\right)$ by an absorbed powerlaw model [photon index $\Gamma=2.14 \pm 0.12$, unabsorbed $F_{\mathrm{X}}=$ $\left.(5.8 \pm 0.4) \times 10^{-13} \mathrm{ergs} \mathrm{cm}^{-2} \mathrm{~s}^{-1}\right]$. The equivalent $\mathrm{H}$ column density, $N_{\mathrm{H}}=(2.2 \pm 0.4) \times 10^{21} \mathrm{~cm}^{-2}$, is marginally consistent with the expected Galactic column density in the source direction, $N_{\mathrm{H}}=1.1 \times 10^{21} \mathrm{~cm}^{-2}$ (Dickey \& Lockman 1990). Examining the X-ray hardness ratio (e.g., Butler \& Kocevski 2007b), there is no evidence for spectral evolution during the XRT observation.

\subsection{KAIT Observations}

KAIT is a $0.76 \mathrm{~m}$ robotic telescope at Lick Observatory that is dedicated to searching for and observing supernovae and monitoring other variable or ephemeral celestial phenomena. It is equipped with a Finger Lakes Instrument (FLI) ProLine PL77 back-illuminated CCD camera having a resolution of $0.8^{\prime \prime} \mathrm{pixel}^{-1}$ and a total field of view (FOV) of $\sim 6.8^{\prime} \times 6.8^{\prime}$. More information on KAIT can be found in Li et al. (2000), Filippenko et al. (2001), and Filippenko (2005) while the KAIT GRB alert system is described in detail by Li et al. (2003b). Notable KAIT observations of GRBs include GRB 021211 ( $\mathrm{Li}$ et al. 2003a), GRB 051111 (Butler et al. 2006), GRB 060210 (Li 2006; W. Li et al. 2008, in preparation), and GRB 080319B (Bloom et al. 2008).

Several improvements have been implemented for the KAIT GRB alert system since the description given by Li et al. (2003b). An FLI PL77 camera has replaced the Apogee AP7 camera, offering a much faster readout time (1.2 $\mathrm{s}$ for FLI vs. $11.0 \mathrm{~s}$ for Apogee). A new feature has been incorporated into the software so the system can easily terminate an ongoing exposure in preparation for the GRB response sequence. Most importantly, a realtime image-processing pipeline has been developed to compare the KAIT images to archival Digital Sky Survey (DSS) images 


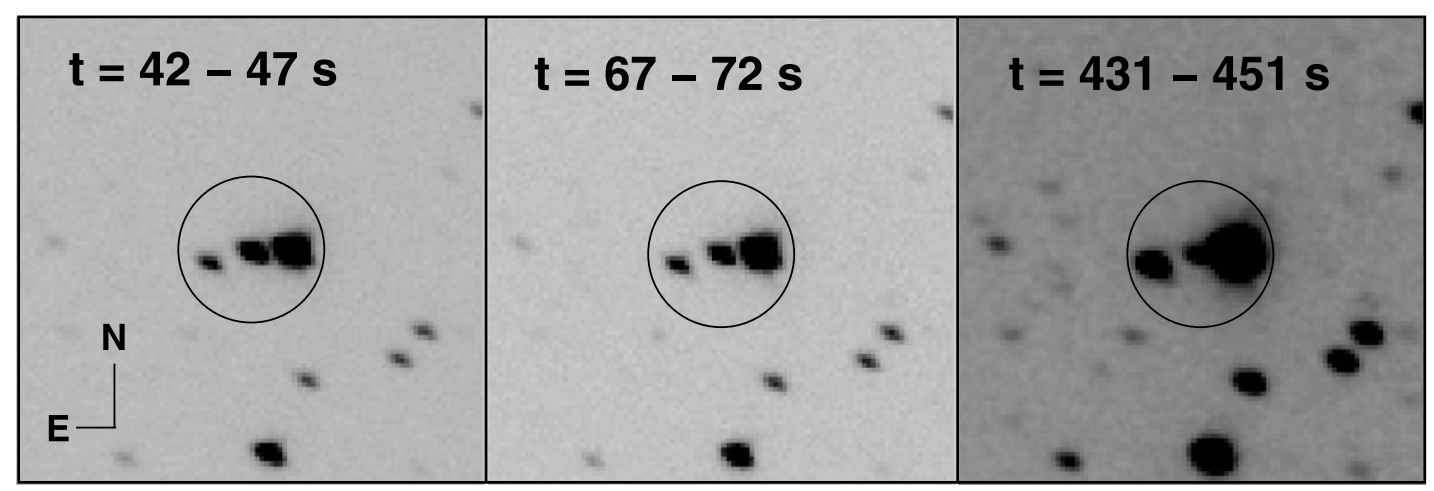

FIG. 2.- Sequence of KAIT images for the OA of GRB 071003. An $80^{\prime \prime} \times 80^{\prime \prime}$ section is shown for the first and fifth unfiltered $5 \mathrm{~s}$ images and for a $20 \mathrm{~s}$ unfiltered image that started at $t=431 \mathrm{~s}$. The $\mathrm{OA}$ is the central object in the circles. It is well detected in the early images and rapidly fades. The image quality is poor owing to the very high air mass of the object.

to identify new objects. Astrometry solutions are derived for the KAIT images by matching the detected objects to the USNO-B1 catalog (Monet et al. 2003), providing coordinates to any new objects to a precision of $\sim 0.2^{\prime \prime}$. Point-spread function (PSF) fitting photometry is also performed on new objects and calibrated to the red magnitudes of the stars in the USNO-B1 catalog. The image-processing results are displayed in real time on a Web site. ${ }^{23}$

For GRB 071003, the KAIT GRB alert program received the GCN socket notice at $t=16 \mathrm{~s}$. The system immediately terminated the ongoing supernova search program and began to slew the telescope to the GRB position. After slewing from close to meridian to an hour angle of $4.2 \mathrm{hr}$, a sequence of $5 \times 5 \mathrm{~s}$ unfiltered images began at $t=42 \mathrm{~s}$. KAIT then switched to a sequence that alternated with $20 \mathrm{~s} V, I$, and unfiltered images. Finally, the sequence converted to $20 \mathrm{~s} I$ and unfiltered images. Because of the physical west hour angle limit of $4.7 \mathrm{hr}$, KAIT only finished part of this prearranged sequence. In total, 56 images were obtained in the $V, I$, and unfiltered passbands from $t=42$ to $1628 \mathrm{~s}$, with full width at half-maximum (FWHM) of $\sim 3^{\prime \prime}$.

Visual inspection of the image-processing results revealed a true new object, first reported by our group ( $\mathrm{Li} 2007)$, measured at 12.8 mag at a position of $\alpha=20^{\mathrm{h}} 07^{\mathrm{m}} 24.12^{\mathrm{s}}, \delta=+10^{\circ} 56^{\prime} 51.8^{\prime \prime}$ (equinox J2000.0; approximate $1 \sigma$ astrometric uncertainty $0.3^{\prime \prime}$ ). Our candidate OA was subsequently confirmed by observations from the automated Palomar 60 inch $(1.5 \mathrm{~m})$ telescope (P60; Cenko \& Fox 2007). Further preliminary analysis of OA earlytime behavior from the KAIT observations was reported by $\mathrm{Li}$ et al. (2007). Figure 2 shows a sequence of the KAIT images for the OA of GRB 071003. An $80^{\prime \prime} \times 80^{\prime \prime}$ section is shown for the first and fifth unfiltered $5 \mathrm{~s}$ image and a $20 \mathrm{~s}$ unfiltered image that started at $t=431 \mathrm{~s}$. As seen in Figure 2 and reported by several groups (Li 2007; Cenko \& Fox 2007; Misra et al. 2007; Li et al. 2007), a bright ( $R \approx 11 \mathrm{mag}$ ) foreground star is located $6.5^{\prime \prime}$ west of the OA of GRB 071003. As discussed in $\S 3$, the presence of this bright star complicates the photometry for the OA, and various methods have been used to minimize its contamination.

\subsection{P60 Observations}

P60 (Cenko et al. 2006) automatically responded to the Swift trigger for GRB 071003, beginning a preprogrammed sequence of observations at 07:43:51 UT (176 s after the trigger). Observations were taken in the Kron $R$, Sloan $i^{\prime}$ and $z^{\prime}$, and Gunn $g$ filters at large air mass $(>2.5)$. Individual images were reduced in

${ }^{23}$ See http://hercules.berkeley.edu/grbdata/grbfinder.gif. real time by our automated reduction pipeline. Manual inspection revealed a fading point source (Cenko \& Fox 2007) in all four filters at the location reported by $\mathrm{Li}$ (2007).

A second epoch of observations was manually scheduled for the night of UT October 4. In an attempt to lessen the contamination of the nearby bright saturated star, these observations were taken in the Johnson $V$-band filter in relatively short (30 s) exposures. A sequence of 30 images was obtained.

\subsection{AEOS Observations}

The $3.6 \mathrm{~m}$ US Air Force Advanced Electro-Optical System (AEOS) telescope, located at the Maui Space Surveillance System on Haleakala, ${ }^{24}$ observed the OA of GRB 071003 with the AEOS Burst Camera (ABC; Flewelling-Swan et al. 2006). ABC has a back-illuminated $2048 \times 2048$ pixel EEV chip, with a scale of $0.189^{\prime \prime} \mathrm{pixel}^{-1}$ and an FOV of $\sim 6.5^{\prime} \times 6.5^{\prime}$. There is no direct internet access to AEOS. After Swift detected the GRB, a fax alert was automatically sent to the AEOS control room, to initiate a series of Target-of-Opportunity ( ToO) observations.

The AEOS observations of GRB 071003 are all unfiltered $10 \mathrm{~s}$ exposures. The first batch of images started at $\sim 9$ minutes after the BAT trigger, and 238 images were observed until $t \approx 83$ minutes, all with very good image quality $\left(\mathrm{FWHM} \approx 0.9^{\prime \prime}\right)$. The second batch of images started at $t \approx 205$ minutes, and 56 images were observed until $t \approx 222$ minutes. Due to the large air mass for these observations and the degraded seeing conditions, however, the images have rather poor quality. We have tried various methods to measure the brightness of the OA in these images but failed. Accordingly, only the first batch of 238 images is analyzed in this study. Preliminary analysis of the AEOS observations is reported by Swan et al. (2007).

\subsection{Keck I/Gemini-S Observations}

In response to the detection of the OA of GRB 071003, we organized a campaign to obtain spectroscopy and late-time photometry with the $10 \mathrm{~m}$ Keck I and the $8 \mathrm{~m}$ Gemini-S telescopes. At $t \approx 2.6 \mathrm{hr}$, we attempted to observe the OA with the HIRES spectrograph at Keck I, but the data are of poor signal-to-noise ratio (S/N) and no obvious lines were detected (Perley et al. 2007b). Just before the HIRES spectroscopy started, we also obtained guider images for the OA, providing important photometric coverage during a gap in the photometry obtained elsewhere (see $\S 3.4)$.

\footnotetext{
${ }^{24}$ Based on data from the Maui Space Surveillance System, which is operated by Detachment 15 of the US Air Force Research Laboratory's Directed Energy Directorate.
} 
The guider images have a scale of $0.37^{\prime \prime}$ pixel $^{-1}$ with an FOV of $53.5^{\prime \prime} \times 71.3^{\prime \prime}$.

On 2007 October 4, we observed the GRB 071003 OA with the Low Resolution Imaging Spectrometer (LRIS; Oke et al. 1995) on Keck I. Anticipating significant fading of the OA, a series of deep $300 \mathrm{~s}$ images was taken with the $g$ and $R$ filters under excellent seeing conditions (FWHM $\left.\approx 0.5^{\prime \prime}\right)$. Inspection of the images reveals that the OA was still bright and saturated in most of the images. Consequently, only a single image in each of the $g$ and $R$ bands, where the OA is not saturated, is analyzed in this study. LRIS uses a beam splitter to separate the light between two arms, red and blue. Both the blue and red cameras have a usable FOV of $\sim 6.0^{\prime} \times 7.8^{\prime}$. The red camera used a back-illuminated Tek $2048 \times 2048$ pixel chip with a scale of $0.215^{\prime \prime}$ pixel $^{-1}$, while the blue camera has a mosaic of two $2048 \times 4096$ pixel Marconi chips with a scale of $0.135^{\prime \prime} \mathrm{pixel}^{-1}$.

Encouraged by the brightness of the OA, we also performed LRIS spectroscopy of the OA. A preliminary analysis of the spectrum is reported by Perley et al. (2007a), and a more detailed analysis is presented in $\S 3.5$.

We performed more LRIS imaging for the OA of GRB 071003 on 2007 October $8,9,10,11$, and 15 , using various combinations of $u, g, V$, and $R$ filters. The presence of the very bright star presents a significant challenge to extracting useful data on the OA, as its diffraction spikes change positions and intensity according to the time and seeing conditions of the observations. Unfortunately, observations on 2007 October 8 were adversely affected by diffraction spikes and poor seeing and were not usable. The data taken on 2007 October 15 are seriously affected by clouds and do not provide an interesting limit to the brightness of the OA, so they are not used in this study.

We also triggered our TOO program (GS-2007B-Q-2; PI H.-W. Chen) for GRBs with the Gemini-S telescope and obtained $g-, r$-, $i$-, and $z$-band images with the GMOS camera on 2007 October 5 and 6. The GMOS camera is equipped with three back-illuminated EEV $2048 \times 4608$ pixel chips. For our observations, the camera is used in a $2 \times 2$ binning mode with a scale of $0.146^{\prime \prime}$ pixel $^{-1}$ and an FOV of $\sim 5.5^{\prime} \times 5.5^{\prime}$. Unfortunately, the 2007 October 5 images are badly affected by bleeding from the very bright star and are not used in this study.

As part of the efforts to follow the evolution of the OA of GRB 071003, we also performed adaptive optics (AO) observations with Keck I on 2007 October 19 (Pollack et al. 2007). The details of the AO observations can be found in $\S 3.6$.

\subsection{Radio Observations}

GRB 071003 was observed with the Very Large Array ${ }^{25}$ (VLA) on various occasions. We made the observation in the B configuration array. We used VLA source $1950+081$ as phase calibrator for $4.86 \mathrm{GHz}$ (C) band observations and 2001+104 for $8.46 \mathrm{GHz}(\mathrm{X})$ band observations. The data were analyzed using standard data reduction routines of the Astronomical Image Processing System (AIPS). The first observation took place on 2007 October 5 in the X band with flux density of $393 \pm 55 \mu \mathrm{Jy}$. Since then we made six observations in the $\mathrm{X}$ band and three observations in the $\mathrm{C}$ band (Table 1).

\section{DATA REDUCTION}

The bright star in the neighborhood of the OA of GRB 071003 makes it a challenge to measure reliable photometry from the data

\footnotetext{
${ }^{25}$ The NRAO is a facility of the National Science Foundation, operated under cooperative agreement by Associated Universities, Inc.
}

TABLE 1

Radio Observations of GRB 071003

\begin{tabular}{|c|c|c|c|c|}
\hline UT Date, Observation & $\begin{array}{l}t_{\mathrm{mid}} \\
(\mathrm{hr})\end{array}$ & $\begin{array}{l}\text { Frequency } \\
\text { (GHz) }\end{array}$ & $\begin{array}{l}\text { Flux Density } \\
\qquad(\mu \mathrm{Jy})\end{array}$ & $\begin{array}{l}\text { Error } \\
(\mu \mathrm{Jy})\end{array}$ \\
\hline 2007 Oct $5,1.85 \ldots$ & 42.168 & 8.46 & 393 & 55 \\
\hline 2007 Oct $7,3.38 \ldots \ldots \ldots \ldots$ & 91.698 & 8.46 & 430 & 50 \\
\hline 2007 Oct $7,3.92 \ldots \ldots \ldots \ldots . .$. & 92.238 & 4.86 & 220 & 54 \\
\hline 2007 Oct $12,1.03 \ldots \ldots \ldots \ldots$ & 209.248 & 8.46 & 431 & 51 \\
\hline 2007 Oct $14,14.84 \ldots \ldots \ldots$ & 271.158 & 8.46 & 332 & 67 \\
\hline 2007 Oct $24,23.58 \ldots \ldots \ldots$ & 519.898 & 8.46 & 260 & 42 \\
\hline 2007 Oct $25,0.04 \ldots \ldots \ldots \ldots$ & 520.358 & 4.86 & 119 & 46 \\
\hline 2007 Nov $5,0.01 \ldots \ldots \ldots \ldots . . .$. & 785.328 & 8.46 & 109 & 45 \\
\hline 2007 Nov $7,0.18 \ldots \ldots \ldots \ldots$ & 833.336 & 4.86 & 93 & 52 \\
\hline
\end{tabular}

described in $\S 2$. In this section we describe the methods used to minimize its contamination.

\subsection{Photometric Calibrations}

For photometric calibrations, the field of GRB 071003 was observed in $B, V, R$, and $I$ on two photometric nights (2007 October 7 and 8) at Lick Observatory, using both KAIT and the Lick Nickel $1 \mathrm{~m}$ telescope. About a dozen Landolt standard-star fields (Landolt 1992) were observed at different air masses throughout each photometric night. Photometric solutions to the Landolt standard stars yield a scatter of $\sim 0.02 \mathrm{mag}$ for all the filters. The GRB 071003 field was also observed for several sets of $B V R I$ images with different depth on both nights. The photometric solutions are used to calibrate a set of local standard stars in the GRB 071003 field. Because the GRB 071003 field is quite crowded, the number of calibrated local standard stars is large (Table 2). A finder for a subset of 23 relatively bright local standard stars is in Figure 3. As seen in Table 2, the local standard stars in the field of GRB 071003 are well calibrated, with standard deviation of the mean (SDOM) of $\sim 0.01 \mathrm{mag}$ for all the BVRI bands. We refer to this calibration as the "Lick calibration" throughout the rest of the paper.

Several Landolt standard-star fields were also observed with LRIS at Keck I: in the $u, g$, and $R$ bands on 2007 October 9, and in the $V$ band on 2007 October 11 . As the number of the observed standard-star fields is small, it is not possible to derive a complete photometric solution for either night. Since the GRB field was observed at similar air masses with some of the standard-star fields, we can treat the LRIS filters as standard and derive the magnitudes for the local standard stars via differential photometry. Unfortunately, this procedure suggests that the 2007 October 9 night was not photometric, as different standard-star observations yield somewhat different zero points. The 2007 October 11 night was photometric, but only the $V$-band standard stars were observed.

We elected to use the Lick calibration as the foundation for all the photometric calibrations, except in the case of the $u$ band. The Lick-calibrated magnitudes are in $B V R I$ and can be reliably converted to the $g, r$, and $i$ bands using color transformation equations (Jester et al. 2005). The conversion to the $z$ band (Rodgers et al. 2006) is somewhat problematic, and as a result we adopt a relatively large uncertainty for the converted magnitudes. For the $u$ band, only two standard-star fields were observed with LRIS on 2007 October 9 , and they give a difference of $0.30 \mathrm{mag}$ in the zero points. We chose to calibrate the GRB 071003 field with the standard-star field that is closer in time of GRB observation, but we added an uncertainty of $0.30 \mathrm{mag}$ to all the calibrated magnitudes. We note that the true error for the $u$-band calibration may 
TABLE 2

Photometry of Comparison Stars in the Field of GRB 071003

\begin{tabular}{|c|c|c|c|c|c|c|c|c|c|c|}
\hline ID & $\begin{array}{c}\alpha_{\mathrm{J} 2000.0} \\
(\operatorname{deg})\end{array}$ & $\begin{array}{c}\delta_{\mathrm{J} 2000.0} \\
(\mathrm{deg})\end{array}$ & $B$ & $N_{B}$ & V & $N_{V}$ & $R$ & $N_{R}$ & $I$ & $N_{I}$ \\
\hline $1 \ldots \ldots \ldots \ldots \ldots$ & 301.9066 & 10.9743 & $16.760(014)$ & 4 & $15.749(012)$ & 4 & $15.235(008)$ & 4 & $14.720(007)$ & 4 \\
\hline 3................ & 301.8926 & 10.9249 & $14.865(008)$ & 9 & $13.243(006)$ & 8 & $12.337(008)$ & 9 & $11.451(008)$ & 7 \\
\hline $4 \ldots \ldots \ldots \ldots$ & 301.8903 & 10.9968 & $15.686(006)$ & 6 & $14.748(009)$ & 8 & $14.255(009)$ & 9 & $13.818(008)$ & 8 \\
\hline $5 \ldots \ldots \ldots \ldots$ & 301.8811 & 10.9343 & $15.026(009)$ & 8 & $13.514(008)$ & 9 & $12.679(007)$ & 10 & $11.882(006)$ & 7 \\
\hline $6 \ldots \ldots \ldots \ldots$ & 301.8799 & 10.9803 & $17.088(009)$ & 7 & $16.050(009)$ & 8 & $15.499(008)$ & 8 & $14.939(010)$ & 6 \\
\hline $9 \ldots \ldots \ldots$ & 301.8693 & 10.9253 & $16.473(009)$ & 8 & $15.631(008)$ & 10 & $15.149(006)$ & 10 & $14.714(008)$ & 9 \\
\hline $10 \ldots \ldots \ldots \ldots$ & 301.8688 & 10.9989 & $15.139(010)$ & 5 & $14.523(009)$ & 6 & $14.153(008)$ & 7 & $13.804(008)$ & 6 \\
\hline $11 \ldots \ldots \ldots \ldots$ & 301.8671 & 10.9417 & $15.310(009)$ & 8 & $14.537(008)$ & 10 & $14.095(007)$ & 10 & $13.692(008)$ & 10 \\
\hline $12 \ldots \ldots \ldots \ldots$ & 301.8570 & 11.0033 & $15.828(012)$ & 5 & $15.004(012)$ & 4 & $14.569(009)$ & 6 & $14.158(010)$ & 4 \\
\hline 13................. & 301.8467 & 10.9749 & $13.470(008)$ & 7 & $12.899(008)$ & 9 & $12.554(008)$ & 10 & $12.220(007)$ & 8 \\
\hline $14 \ldots \ldots \ldots \ldots$ & 301.8396 & 10.9967 & $16.468(009)$ & 7 & $15.752(008)$ & 9 & $15.331(007)$ & 10 & $14.936(009)$ & 9 \\
\hline $19 \ldots \ldots \ldots \ldots$ & 301.8334 & 10.9963 & $16.472(012)$ & 6 & $15.430(010)$ & 7 & $14.786(008)$ & 10 & $14.254(008)$ & 9 \\
\hline $20 \ldots \ldots \ldots \ldots$ & 301.8311 & 11.0048 & $16.227(012)$ & 4 & $15.529(009)$ & 3 & $15.056(007)$ & 6 & $14.631(006)$ & 5 \\
\hline $21 \ldots \ldots \ldots \ldots \ldots$ & 301.8279 & 10.9884 & $17.045(008)$ & 7 & $16.107(008)$ & 9 & $15.546(008)$ & 10 & $15.007(009)$ & 8 \\
\hline $22 \ldots \ldots \ldots \ldots$ & 301.8247 & 10.9781 & $14.276(008)$ & 9 & $13.825(008)$ & 9 & $13.510(008)$ & 10 & $13.223(008)$ & 9 \\
\hline $23 \ldots \ldots \ldots \ldots \ldots$ & 301.8221 & 10.9256 & $16.606(009)$ & 9 & $16.002(012)$ & 6 & $15.623(007)$ & 9 & $15.204(010)$ & 7 \\
\hline
\end{tabular}

Notes.-Uncertainties (standard deviation of the mean) are indicated in parentheses. Table 2 is published in its entirety in the electronic edition of the Astrophysical Journal. A portion is shown here for guidance regarding its form and content.

be higher than 0.30 mag due to the nonphotometric conditions on 2007 October 9.

\subsection{KAIT Data Reduction}

The KAIT data were automatically processed with bias and dark current subtraction and flat-fielding. The PSF of the OA is

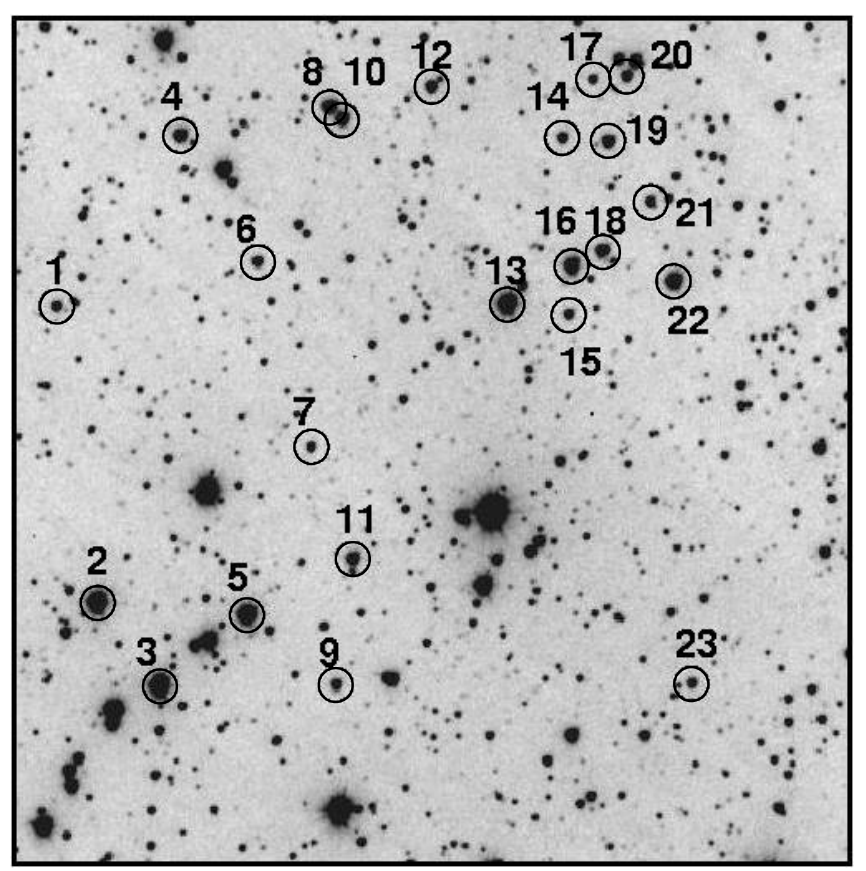

FIG. 3.-Finder chart for a subset of local standard stars in the field of GRB 071003. The field of view is $6.6^{\prime} \times 6.6^{\prime}$. North is up and east is to the left. The displayed image is the unfiltered template taken with KAIT on 2007 October 9. seriously affected by the bright star, which is less than 10 pixels away in the KAIT images. Consequently, normal PSF-fitting photometry cannot fit the peak and background of the OA simultaneously to produce a reliable measurement.

We use image subtraction to remove the contamination of the bright star. To generate template images for subtraction, KAIT imaged the GRB 071003 field in the unfiltered mode and in the $V$ and $I$ filters for the next several nights after the burst. To make sure the bright star is not saturated, short $(5 \mathrm{~s})$ exposures were used, and $50-100$ images for each filter were acquired to ensure high $\mathrm{S} / \mathrm{N}$ in the combined images. As discussed in $\S 4$, the GRB OA was still reasonably bright in the second night after the burst, so we used the images obtained at 4-6 days after the burst as the template for the field without significant OA contribution. Our image subtraction code is based on the ISIS package (Alard \& Lupton 1998) as modified by B. Schmidt for the High- $z$ Supernova Search Team (Schmidt et al. 1998). An illustration of the image subtraction is presented in the top panels of Figure 4.

The Lick calibration was used to transform the KAIT instrumental magnitudes to the standard Johnson $V$ and Cousins $I$ passbands, with proper color terms measured from the photometric nights. We also find that the combination of the KAIT optics and the quantum efficiency of the FLI CCD camera makes the KAIT unfiltered observations mostly mimic the $R$ band. During the two photometric nights, unfiltered observations of the Landolt standard-star fields were also performed. Analysis of these images indicates that the KAIT unfiltered magnitudes can be effectively transformed to the $R$ band, with a relatively large color term and an rms of $\sim 0.05 \mathrm{mag}$, similar to the earlier results we reported ( $\mathrm{Li}$ et al. 2003a, 2003b; Butler et al. 2006).

To increase the $\mathrm{S} / \mathrm{N}$, the late-time KAIT images of GRB 071003 were combined into groups of three to eight images. The final KAIT photometry for the GRB 071003 OA is listed in Table 3. The reported error bars are the uncertainties in PSF-fitting photometry 


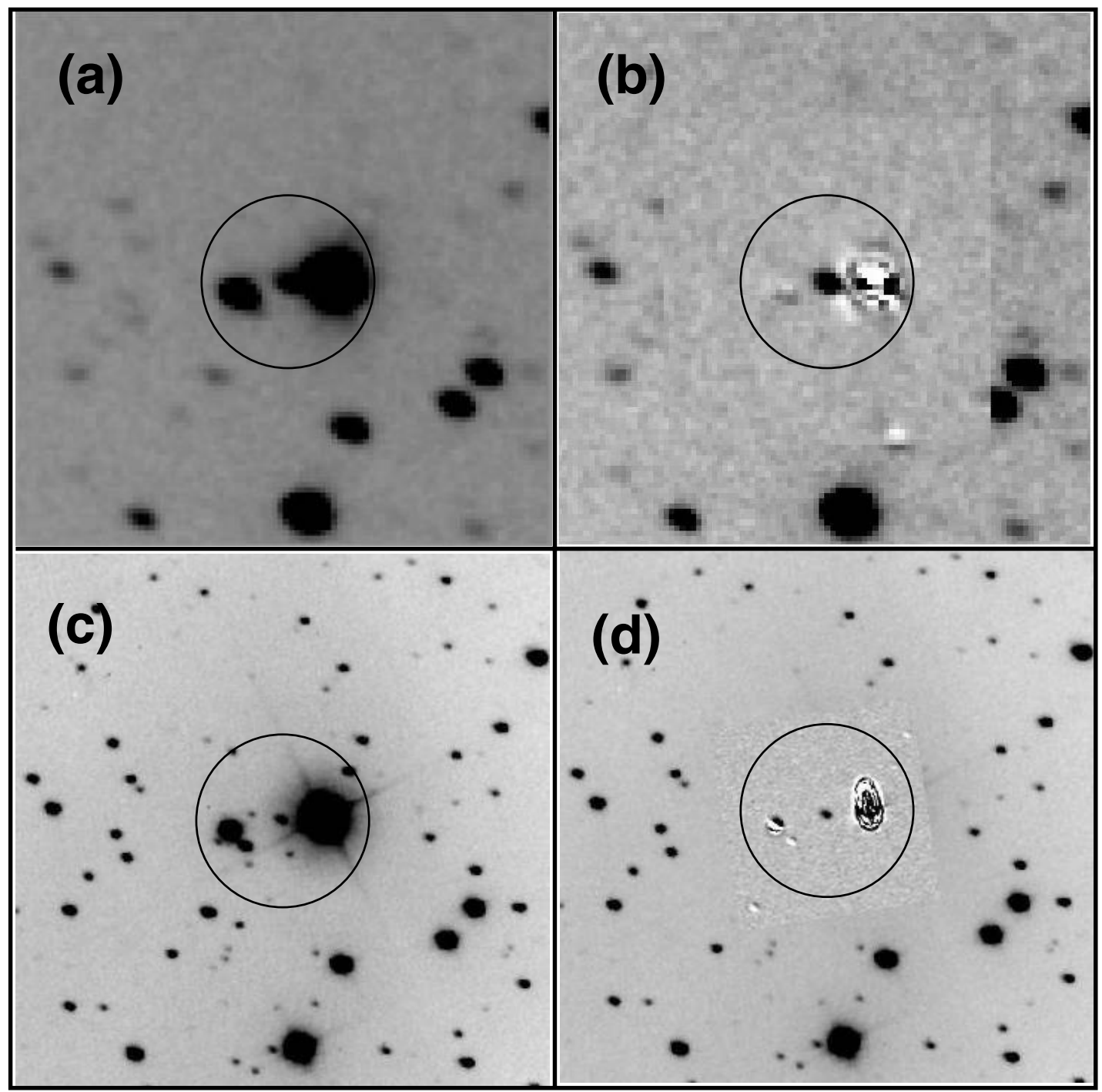

FIG. 4.- Illustration of using image subtraction to remove the contamination of the bright nearby star to the OA of GRB 071003 . The KAIT image subtraction code is demonstrated here. (a) An $80^{\prime \prime} \times 80^{\prime \prime}$ section of the original $20 \mathrm{~s}$ unfiltered KAIT image of the OA taken at $t=431 \mathrm{~s} ;(b)$ the same section after image subtraction of the central $50^{\prime \prime} \times 50^{\prime \prime}$ using an unfiltered template image after the OA has faded; $(c)$ an $80^{\prime \prime} \times 80^{\prime \prime}$ section of the combined unfiltered AEOS image at $t=5002.6 \mathrm{~s}$; and $(d)$ the same section after image subtraction of the central $30^{\prime \prime} \times 30^{\prime \prime}$ using a hand-made template image. See text for more details.

and those in the calibration process, added in quadrature. A plot of the KAIT photometry, along with measurements from other telescopes during the same time span (with BAT data overplotted and fitted by a chromatic model described in $\S 4.3$ ), is presented in Figure 5.

\subsection{AEOS Data Reduction}

The ABC images were processed using dark subtraction only. Because of highly variable stray light and vignetting, we did not apply a flat field to these images. We used SExtractor (Bertin \& Arnouts 1996) to find all the sources in the images, from which we were able to determine the astrometry.

We employed the NN2 flux difference method (Barris et al. 2005; hereafter the NN2 method) for constructing the AEOS light curve. The NN2 method also uses image subtraction to measure the fluxes for a variable source, but it does not designate one particular image as the template. Instead, given $N$ total observations, the NN2 method solves for the vector of fluxes from the individual images using the antisymmetric matrix of flux differences from the $N(N-1) / 2$ distinct possible subtractions. Compared to the template image subtraction method, the NN2 method takes all the available information from the images into account and is less susceptible to possible noise associated with a single template image. To avoid a large number of image subtractions, we combined the original 238 AEOS observations into 39 images. For the first 228 images, each set of six consecutive images is combined into one. The last 10 images are combined into a single image. We compared the results from the NN2 method to those from a traditional template image subtraction method (Fig. 4, bottom) and found them to be consistent with each other.

To calibrate the AEOS data to the standard photometry system, we used the KAIT $R$-band data during the overlap period and assume that the unfiltered AEOS data have no color term to the $R$ band. ${ }^{26}$ The final AEOS photometry is listed in Table 4. The reported error bars are only those output by the NN2 method and do not include a possible large systematic error due to calibration. If the throughput of the AEOS telescope in the unfiltered

26 We attempted to quantify the color term of the unfiltered AEOS data to the standard $R$ system using the local standard stars in the field of GRB 071003 but found no apparent correlation between the scatter of the (unfiltered $-R$ ) differences and the colors of the stars. 
TABLE 3

KAIT РнотомETRY OF GRB 071003

\begin{tabular}{|c|c|c|c|c|}
\hline $\begin{array}{l}t_{\text {start }}{ }^{\mathrm{a}} \\
(\mathrm{s})\end{array}$ & $\begin{array}{c}\text { Exposure Time } \\
\text { (s) }\end{array}$ & Magnitude & Error & Filter \\
\hline $42.0 \ldots \ldots \ldots \ldots \ldots \ldots$ & 5 & 12.791 & 0.019 & $R^{\mathrm{b}}$ \\
\hline $49.0 \ldots \ldots \ldots \ldots \ldots \ldots \ldots$ & 5 & 12.999 & 0.024 & $R$ \\
\hline $55.0 \ldots \ldots \ldots \ldots \ldots \ldots \ldots \ldots \ldots \ldots \ldots \ldots \ldots$ & 5 & 13.193 & 0.021 & $R$ \\
\hline 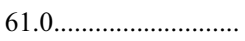 & 5 & 13.321 & 0.024 & $R$ \\
\hline $67.0 \ldots \ldots \ldots \ldots$. & 5 & 13.500 & 0.019 & $R$ \\
\hline 97.0...... & 20 & 14.465 & 0.027 & V \\
\hline 128.0 & 20 & 13.919 & 0.032 & $I$ \\
\hline $157.0 \ldots \ldots \ldots \ldots \ldots \ldots \ldots$ & 20 & 14.382 & 0.031 & $R$ \\
\hline $188.0 \ldots \ldots \ldots \ldots \ldots \ldots \ldots$ & 20 & 14.916 & 0.034 & $V$ \\
\hline $219.0 \ldots \ldots$ & 20 & 14.121 & 0.022 & $I$ \\
\hline $249.0 \ldots \ldots \ldots$ & 20 & 14.750 & 0.023 & $R$ \\
\hline 279.0 & 20 & 15.409 & 0.035 & $V$ \\
\hline $310.0 \ldots \ldots \ldots \ldots \ldots \ldots \ldots$ & 20 & 14.578 & 0.030 & $I$ \\
\hline $340.0 \ldots \ldots \ldots \ldots \ldots \ldots \ldots$ & 20 & 15.401 & 0.024 & $R$ \\
\hline $370.0 \ldots \ldots$ & 20 & 16.034 & 0.107 & $V$ \\
\hline 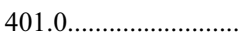 & 20 & 15.478 & 0.063 & $I$ \\
\hline $431.0 \ldots \ldots \ldots \ldots \ldots$ & 20 & 16.239 & 0.082 & $R$ \\
\hline 462.0 & 20 & 16.853 & 0.200 & $V$ \\
\hline 492.0 & 20 & 15.977 & 0.069 & $I$ \\
\hline $522.0 \ldots \ldots \ldots \ldots \ldots \ldots$ & 20 & 16.749 & 0.091 & $R$ \\
\hline $565.0 \ldots \ldots \ldots \ldots$ & 20 & 16.255 & 0.077 & $I$ \\
\hline 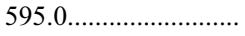 & 20 & 16.849 & 0.097 & $R$ \\
\hline 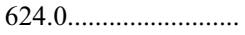 & 20 & 16.364 & 0.106 & $I$ \\
\hline 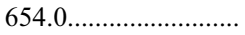 & 20 & 17.041 & 0.089 & $R$ \\
\hline $749.09^{\mathrm{c}}$ & $3 \times 20$ & 16.830 & 0.113 & $I$ \\
\hline $787.68^{\mathrm{c}} \ldots \ldots \ldots \ldots \ldots \ldots$ & $3 \times 20$ & 17.362 & 0.121 & $R$ \\
\hline $1007.74^{\mathrm{c}} \ldots \ldots \ldots \ldots \ldots \ldots$ & $6 \times 20$ & 17.314 & 0.148 & $I$ \\
\hline $1009.95^{\mathrm{c}} \ldots \ldots \ldots \ldots$ & $5 \times 20$ & 17.711 & 0.147 & $R$ \\
\hline $1422.34^{\mathrm{c}} \ldots \ldots \ldots \ldots \ldots$ & $8 \times 20$ & 18.103 & 0.154 & $R$ \\
\hline $1464.58^{\mathrm{c}}$ & $7 \times 20$ & 17.473 & 0.135 & $I$ \\
\hline
\end{tabular}

${ }^{\text {a }}$ The start time of the exposure, in seconds after the BAT trigger.

b The $R$-band photometry is derived from unfiltered observations.

$c$ The time (s) at the middle point of several combined images.

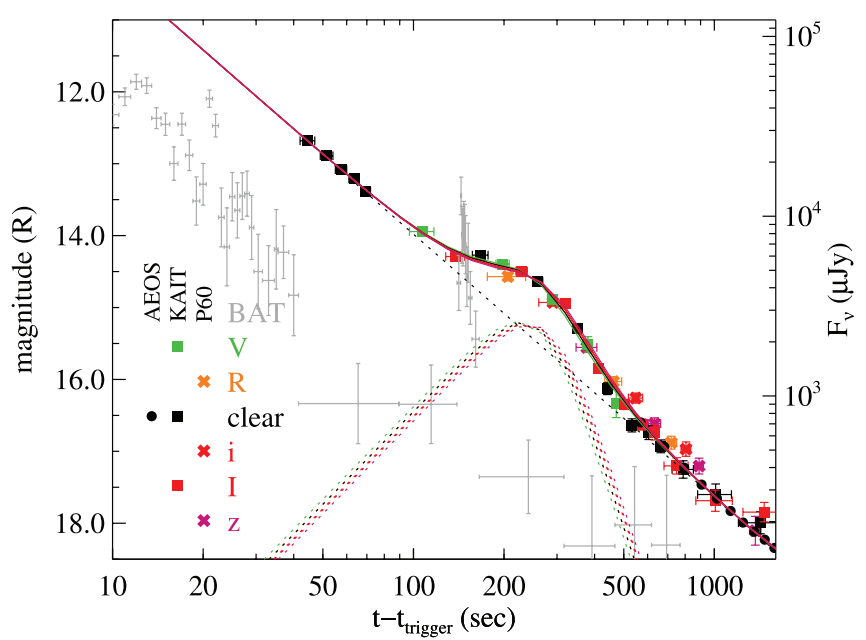

FIG. 5.-Early-time light curve of the optical afterglow of GRB 071003 using KAIT photometry, supplemented by observations from P60 and AEOS. The gammaray light curve from the BAT is overplotted in gray (scaled arbitrarily). A clearly additive "bump" at 100-500 s is apparent. Photometric follow-up observations continued after $2000 \mathrm{~s}$ with P60 and AEOS, as well as with Gemini and Keck in subsequent nights; the complete 16 day optical light curve is presented in Fig. 10.
TABLE 4

AEOS $R$-Band Photometry from Unfiltered Observations

\begin{tabular}{|c|c|c|}
\hline $\begin{array}{c}t_{\mathrm{mid}}{ }^{\mathrm{a}} \\
(\mathrm{s})\end{array}$ & $R$ & $\sigma_{R}$ \\
\hline $568.6 \ldots .$. & 16.708 & 0.016 \\
\hline $681.3 \ldots$ & 17.046 & 0.018 \\
\hline $794.0 \ldots \ldots$. & 17.337 & 0.020 \\
\hline $906.8 \ldots$. & 17.573 & 0.020 \\
\hline $1019.6 \ldots$ & 17.766 & 0.020 \\
\hline $1132.3 \ldots \ldots \ldots$ & 17.940 & 0.020 \\
\hline $1245.1 \ldots \ldots \ldots$ & 18.101 & 0.020 \\
\hline $1357.9 \ldots \ldots$ & 18.229 & 0.023 \\
\hline 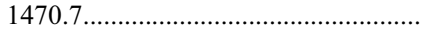 & 18.339 & 0.025 \\
\hline 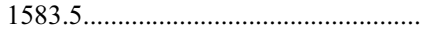 & 18.454 & 0.026 \\
\hline $1696.3 \ldots \ldots$. & 18.545 & 0.028 \\
\hline $1809.1 \ldots \ldots \ldots \ldots . . . .$. & 18.640 & 0.034 \\
\hline ㄴ. & 18.724 & 0.033 \\
\hline $2034.8 \ldots$ & 18.814 & 0.040 \\
\hline $2147.6 \ldots$ & 18.865 & 0.037 \\
\hline $2260.3 \ldots$ & 18.924 & 0.042 \\
\hline . & 18.941 & 0.048 \\
\hline 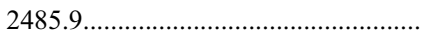 & 18.941 & 0.064 \\
\hline 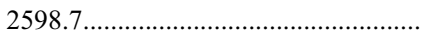 & 19.042 & 0.044 \\
\hline 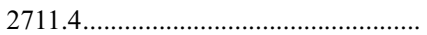 & 19.087 & 0.049 \\
\hline $2824.2 \ldots \ldots \ldots \ldots \ldots$ & 19.104 & 0.050 \\
\hline $2937.0 \ldots \ldots \ldots$ & 19.109 & 0.054 \\
\hline 3049.7 & 19.142 & 0.054 \\
\hline $3162.5 \ldots$ & 19.150 & 0.052 \\
\hline $3275.3 \ldots \ldots \ldots$ & 19.160 & 0.056 \\
\hline 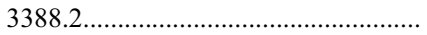 & 19.169 & 0.055 \\
\hline $3501.0 \ldots \ldots \ldots \ldots$ & 19.158 & 0.050 \\
\hline $3613.8 \ldots \ldots \ldots \ldots . . . .$. & 19.139 & 0.056 \\
\hline (1) & 19.185 & 0.055 \\
\hline 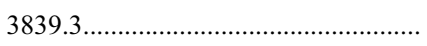 & 19.206 & 0.056 \\
\hline 4062.4 & 19.188 & 0.056 \\
\hline 4175.3 & 19.196 & 0.055 \\
\hline 4288.1 & 19.194 & 0.057 \\
\hline 4401.0 & 19.208 & 0.051 \\
\hline (n) & 19.167 & 0.054 \\
\hline 4626.6 & 19.150 & 0.053 \\
\hline 4739.4 & 19.163 & 0.065 \\
\hline $4852.2 \ldots \ldots \ldots-1$. & 19.160 & 0.057 \\
\hline $5002.6 \ldots \ldots \ldots$ & 19.145 & 0.050 \\
\hline
\end{tabular}

NoтE.-The original data set was grouped and combined into 39 images.

${ }^{\text {a }}$ The time at the middle point of several combined images.

mode is not drastically different from that of KAIT, we estimate the systematic error to be $\sim 0.07$ mag when the GRB OA was bright $(t<20$ minutes) and $\sim 0.15 \mathrm{mag}$ when the GRB became faint ( $t>40$ minutes). The systematic errors can be much higher if the unfiltered throughput is very different for the two telescopes.

\subsection{Keck I/Gemini-S Data Reduction}

Due to the large aperture of the Keck I and Gemini-S telescopes, the bright star close to the GRB 071003 OA produces numerous diffraction spikes, as well as two large blooming spikes along the readout direction. Because the orientation, width, and intensity of the spikes change with the seeing conditions, the exposure duration, and the time of the observations, it is difficult to cleanly remove them using the template image subtraction or the NN2 method. However, due to the high resolution of these images, the spikes are well sampled and show distinct axial symmetry. We developed a saturation spike subtraction method, in which we divide the image of the bright star in half, flip the right 
TABLE 5

Keck/Gemini-S Photometry of GRB 071003

\begin{tabular}{|c|c|c|c|c|c|}
\hline $\begin{array}{c}t_{\mathrm{mid}}{ }^{\mathrm{a}} \\
(\mathrm{s})\end{array}$ & $\begin{array}{c}\text { Exposure Time } \\
\text { (s) }\end{array}$ & Magnitude & Error & Filter & Telescope \\
\hline 9523.7.................... & $2 \times 20$ & $18.59^{\mathrm{b}}$ & 0.25 & $C_{R}$ & Keck I \\
\hline 76891.8.................. & 300 & 20.32 & 0.07 & $g$ & Keck I \\
\hline 77044.0................ & 300 & 19.43 & 0.06 & $R$ & Keck I \\
\hline $231174.7 \ldots \ldots \ldots \ldots$ & 450 & 22.33 & 0.20 & $g$ & Gemini-S \\
\hline $231802.3 \ldots \ldots \ldots \ldots$ & 450 & 21.57 & 0.32 & $i$ & Gemini-S \\
\hline $232430.8 \ldots \ldots \ldots \ldots$ & 450 & 21.97 & 0.22 & $r$ & Gemini-S \\
\hline $233056.5 \ldots \ldots \ldots \ldots$ & 450 & 21.35 & 0.40 & $z$ & Gemini-S \\
\hline $515855.0 \ldots \ldots \ldots \ldots$ & 1485 & 22.61 & 0.30 & $R$ & Keck I \\
\hline $516250.5 \ldots \ldots \ldots \ldots$ & 975 & 23.56 & 0.30 & $g$ & Keck I \\
\hline $517510.4 \ldots \ldots \ldots \ldots$ & 720 & 23.56 & 0.45 & $u$ & Keck I \\
\hline 604978.0.............. & 660 & 23.06 & 0.50 & $R$ & Keck I \\
\hline $605144.4 \ldots \ldots \ldots \ldots$ & 780 & 24.05 & 0.40 & $g$ & Keck I \\
\hline $682211.1 \ldots \ldots \ldots \ldots$ & 330 & 23.56 & 0.40 & V & Keck I \\
\hline $682211.9 \ldots \ldots \ldots \ldots$ & 660 & 24.42 & 0.50 & $u$ & Keck I \\
\hline $682940.0 \ldots \ldots \ldots \ldots$ & 840 & 23.40 & 0.60 & $R$ & Keck I \\
\hline $1373589 \ldots \ldots \ldots \ldots$ & 1800 & 21.58 & 0.03 & $K^{\prime}$ & Keck II \\
\hline
\end{tabular}

a The time at the middle point of the observations.

${ }^{\mathrm{b}}$ Measured from unfiltered images from the Keck I HIRES guider.

side, and subtract it from the left side. Due to the symmetry in the spikes, this subtraction process leaves a reasonably clean region around the GRB OA. PSF-fitting photometry was then performed on the GRB OA in the spike-subtracted images and on a series of local standard stars. The Lick calibration is used to calibrate the Keck I and Gemini-S instrumental magnitudes to the standard system.

The final Keck I and Gemini-S photometry is reported in Table 5. The error bars of the magnitudes are the uncertainties from the PSF-fitting photometry and those in the calibration process added in quadrature. One special data point is the Keck I HIRES guider image at $t=9523.7 \mathrm{~s}$ because it bridges the early KAIT/AEOS data to the late-time Keck I and Gemini-S observations. The GRB OA was well detected in the guider image, but because the image has a small FOV and is unfiltered, photometric calibration becomes particularly difficult.

We have used three methods to calibrate the measured instrumental magnitude of the OA after the guider images were processed with the saturation spike subtraction method: differential photometry between the AEOS unfiltered data and the guider images, photometric calibration to about half a dozen stars in the HIRES guider images using the KAIT unfiltered images, and photometric calibration to these stars using the Keck I $R$-band images. The measured $R$-band magnitudes from these three methods show a scatter of $\sim 0.25 \mathrm{mag}$, and their average value and uncertainty are listed in Table 5.

\subsection{P60 Data Reduction}

The P60 data reduction is presented in this section because it employs several methods (illustrated in Fig. 4) discussed earlier in the paper. We obtained template images for the field after the OA of GRB 071003 has faded. However, the saturation spikes of the bright star close to the GRB ruined the template images in the $R$ and $i^{\prime}$ bands, so we were only able to run image subtraction for the data in the $g$ and $z^{\prime}$ bands. We also employed the saturation spike subtraction methods as described in $\S 3.4$. Although P60 does not have the resolution of the Keck I and Gemini-S telescopes, subtraction of half of the saturation spikes helped to clean up the background of the OA considerably.
TABLE 6

P60 Рhotometry of GRB 071003

\begin{tabular}{|c|c|c|c|c|}
\hline $\begin{array}{l}t_{\mathrm{start}}^{\mathrm{a}} \\
(\mathrm{s})\end{array}$ & $\begin{array}{l}\text { Exposure Time } \\
\text { (s) }\end{array}$ & Magnitude & Error & Filter \\
\hline $176.0 \ldots \ldots \ldots \ldots \ldots \ldots \ldots$ & 60 & 14.57 & 0.06 & $R$ \\
\hline 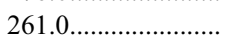 & 60 & 15.08 & 0.05 & $i^{\prime}$ \\
\hline 347.0 ............................ & 60 & 15.37 & 0.07 & $z^{\prime}$ \\
\hline $432.0 \ldots \ldots \ldots \ldots \ldots \ldots \ldots \ldots \ldots \ldots \ldots \ldots$ & 60 & 16.03 & 0.07 & $R$ \\
\hline $518.0 \ldots \ldots \ldots \ldots \ldots \ldots \ldots$ & 60 & 16.41 & 0.07 & $i^{\prime}$ \\
\hline 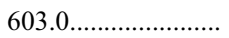 & 60 & 16.43 & 0.08 & $z^{\prime}$ \\
\hline $689.0 \ldots \ldots \ldots \ldots \ldots \ldots \ldots$ & 60 & 16.88 & 0.09 & $R$ \\
\hline $775.0 \ldots \ldots \ldots \ldots \ldots \ldots \ldots \ldots$ & 60 & 17.12 & 0.10 & $i^{\prime}$ \\
\hline $860.0 \ldots \ldots \ldots \ldots \ldots \ldots \ldots$ & 60 & 17.02 & 0.11 & $z^{\prime}$ \\
\hline 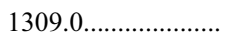 & 120 & 17.92 & 0.20 & $z^{\prime}$ \\
\hline $1454.0 \ldots \ldots \ldots \ldots \ldots$ & 120 & 18.71 & 0.10 & $g$ \\
\hline 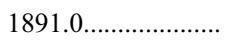 & 120 & 18.67 & 0.28 & $z^{\prime}$ \\
\hline $2037.0 \ldots \ldots \ldots \ldots \ldots$ & 120 & 19.33 & 0.15 & $g$ \\
\hline $2618.0 \ldots \ldots \ldots \ldots$ & 120 & 19.44 & 0.20 & $g$ \\
\hline
\end{tabular}

${ }^{\text {a }}$ The start time of the exposure, in seconds after the BAT trigger.

We also applied a third method to reduce the P60 data. Due to the richness of stars in the GRB 071003 field and the large field of view of the P60 camera $\left(12.9^{\prime} \times 12.9^{\prime}\right)$, we were able to pick a star that is close in brightness (within $0.1 \mathrm{mag}$ in all filters) and thus has similar saturation spikes to the bright star close to GRB 071003. The chosen star is located at $\alpha=20^{\mathrm{h}} 07^{\mathrm{m}} 14.84^{\mathrm{s}}, \delta=$ $+10^{\circ} 53^{\prime} 59.8^{\prime \prime}$ (equinox J2000.0), which is $136.7^{\prime \prime}$ west and $172.0^{\prime \prime}$ south of the GRB 071003 OA. By slightly scaling the PSF of this bright star and subtracting it from the star close to the GRB, we were able to largely remove the complicated background around the GRB OA.

PSF-fitting photometry is applied to the images after different ways of image subtraction, and the Lick calibration is used to calibrate the instrumental magnitudes into the standard system. The final photometry from the P60 data is listed in Table 6, which is the average of the spike and bright star subtraction methods. The results from the template image subtraction method are not considered because the method can only be applied to a subset of filters, but they are consistent with the other two methods within measurement uncertainties.

\subsection{Keck AO Data Reduction}

On 2007 October 19 (starting at UT 05:14) we observed the GRB 071003 OA with the NIRC2 (van Dam et al. 2004) narrowfield camera $\left(0.01^{\prime \prime}\right.$ pixel $\left.^{-1}\right)$ on Keck II using natural guide star adaptive optics (NGS AO). While the extremely bright nearby star greatly complicated the optical analysis, it was ideal to be used as the natural guide star during NGS AO imaging. We took 15 science exposures, each of $60 \mathrm{~s}$ and two co-additions, resulting in a total integration time of 30 minutes. The images were reduced using standard techniques, including dark subtracting, flat-fielding, and filtering for deviant pixels. Each frame was dewarped using the recommended method for NIRC2, and the resulting images were registered to a common origin and combined.

The GRB OA is well detected 2 weeks after the burst, as shown in the final combined image in Figure 6. To measure the brightness of the OA, we created a model of the PSF using shortexposure, unsaturated images of a nearby Two Micron All Sky Survey (2MASS) star $\left(K_{s}=12.011 \pm 0.024 \mathrm{mag}, d=7.8^{\prime \prime}\right)$, taken immediately prior to the science exposures. We then subtracted this model PSF from the OA. With the same 2MASS star as the photometric calibrator, we measure the OA to have 


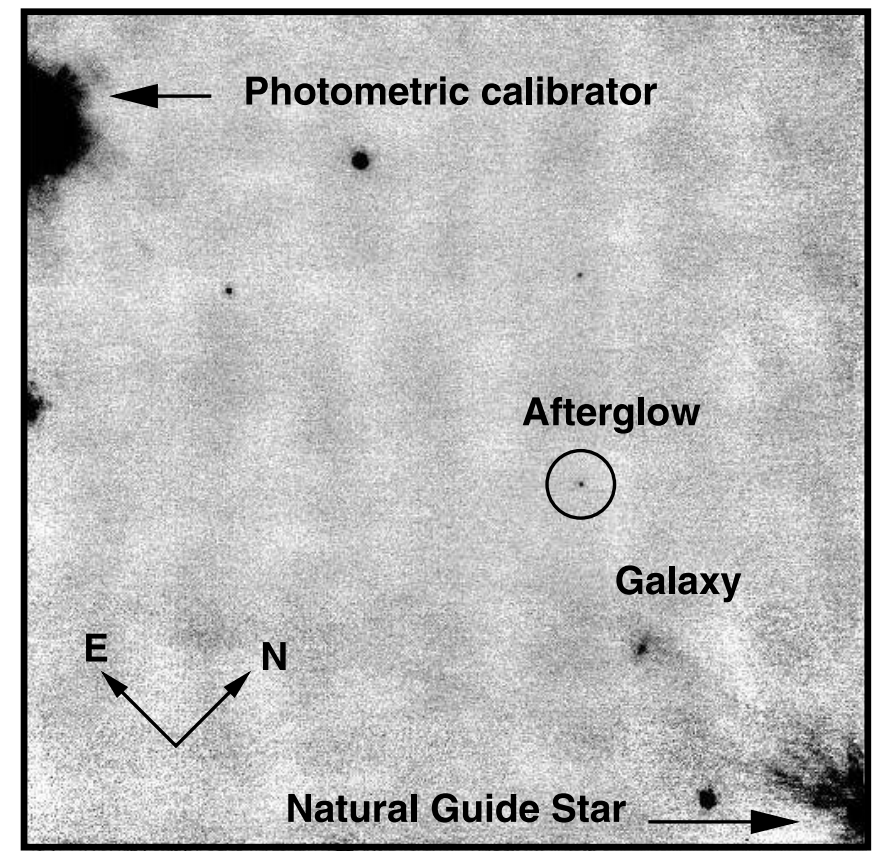

FIG. 6.-NGS AO image of the GRB 071003 field taken with Keck II on 2007 October 19, 16 days after the burst. The FOV is approximately $12^{\prime \prime} \times 10^{\prime \prime}$. The afterglow is well detected with $K^{\prime}=21.58 \pm 0.03 \mathrm{mag}$. No host galaxy emission is detected.

$K^{\prime}=21.65 \pm 0.10$ Vega mag. (Galactic reddening of $A_{K^{\prime}} \approx$ $0.05 \mathrm{mag}$ is negligible along this sight line and has not been applied.)

\subsection{Keck LRIS Spectroscopy Reduction}

We obtained low-resolution optical spectroscopy of the optical afterglow of GRB 071003 on 2007 October 4.335 using the LRIS on the Keck I telescope. A pair of $600 \mathrm{~s}$ dithered exposures was taken under clear conditions at air mass 1.2 with $0.6^{\prime \prime}$ seeing. We used both the blue and red arms of LRIS, with the light split by the D680 dichroic. The 300/5000 grism on the blue side gave a spectral resolution of $8.4 \AA$ over the range $3300-6500 \AA$. We used the $600 / 10000$ grating to achieve $4.1 \AA$ resolution over the range $6500-8630 \AA$. The spectrophotometric standard star Feige 110 (Stone 1977) was observed the following night in the same setup. Intermittent clouds were present the night of the standardstar observation, so the absolute flux scale is unreliable.

The long, $1.0^{\prime \prime}$ wide slit was oriented at a position angle of $10^{\circ}$ for the afterglow observations, which was not the parallactic angle (Filippenko 1982). However, the Cassegrain Atmospheric Dispersion Compensator module (Phillips et al. 2006) was mounted, so the derived spectral shape should be reliable. The exception is in the spectral range of 6000-6500 $\AA$, where second-order blue light contamination is prominent in the spectrum of the standard star. An attempt was made to correct for the contamination, but the spectral slope in this section is more uncertain than in the rest of the spectrum. We also fitted an extinction-corrected power law to the flux-calibrated spectrum (excluding line and secondorder contaminated regions) in an attempt to estimate the spectral slope, but the estimated slope of $f_{\nu} \propto \nu^{-0.87}$ differs significantly from the spectral slope estimated from multiband late-time photometry $(\S 4.7)$. This may be due to continuum contamination from the nearby star in the spectrum (which is difficult to properly remove), so we do not further consider this spectroscopic spectral index.

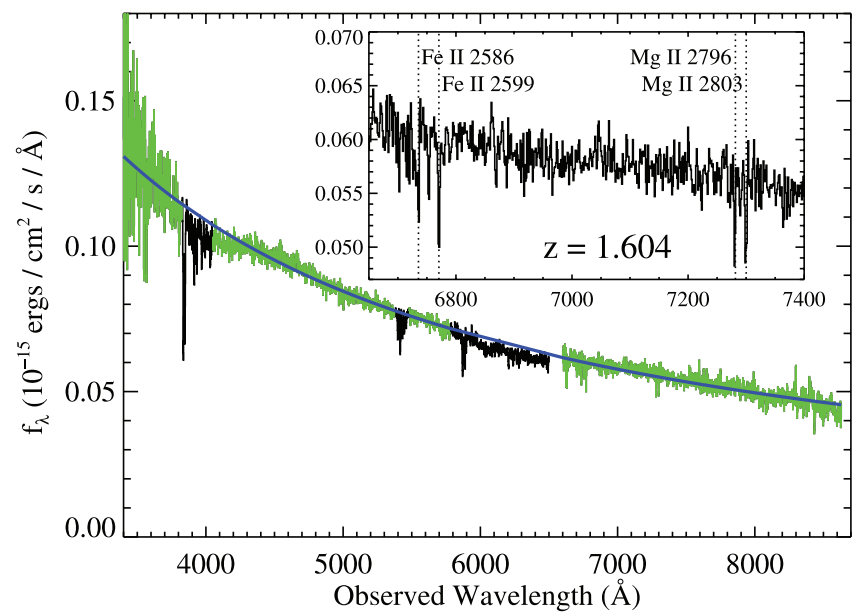

FIG. 7.- Spectrum of the GRB 071003 afterglow covering the full observed spectral range. The spectrum has been flux-calibrated and corrected for Galactic reddening of $E(B-V)=0.148 \mathrm{mag}$. The inset shows an expanded view of the region surrounding the $\mathrm{Fe}$ and $\mathrm{Mg}$ absorption system at the burst redshift. A power-law continuum was fitted to the regions of the spectrum shown in green, chosen to avoid strong absorption lines and the wavelength range contaminated by secondorder blue light. The thick solid blue line shows the resultant fit $\left(f_{\lambda} \propto \lambda^{-1.13}\right.$, or $\left.f_{\nu} \propto \nu^{-0.87}\right)$, but it differs in slope from our more reliable fit to the broadband photometry; thus, it is used only to normalize the spectrum.

The largely featureless spectrum (Fig. 7) has an $\mathrm{S} / \mathrm{N}>5$ pixel $^{-1}$ down to $\sim 3500 \AA$. There is no apparent absorption by the intergalactic medium at these wavelengths, yielding an upper limit to the redshift of the burst of $z_{\mathrm{GRB}}<(3500 / 1216)-1=1.88$. Numerous metal-line absorption lines (but no emission lines) are visible in the spectrum. We have fitted the equivalent widths of all $\gtrsim 5 \sigma$ features in the normalized spectrum using a Gaussian profile and report the rest-frame values in Table 7.

We previously presented (Perley et al. 2007a) analysis of this spectrum, identifying Mg II absorption systems at $z=0.372$ and 1.100. A VLT spectrum acquired the same night (Fugazza et al. 2007 ) identified a third absorption system at $z=0.937$, which is confirmed by our observations. These are the only strong absorption systems in the data, and previously we considered it likely that the $z=1.100$ system originated from the host galaxy (Fig. 8). Surprisingly, however, a more thorough investigation revealed a fourth, weak absorption system at a higher redshift of $z=1.604$ (Fig. 9). Contrary to our expectation, the gas at this redshift has the weakest Mg II absorption of the four systems.

This is remarkable: absorption lines associated with GRB environments are generally very strong with rest-frame equivalent widths exceeding several angstroms (Savaglio et al. 2003; Prochaska et al. 2008). Figure 8 also indicates, however, the presence of finestructure $\mathrm{Fe}$ II transitions at this redshift. With the exception of active galactic nucleus environments, these transitions have only been identified in gas surrounding the GRB phenomenon (Prochaska et al. 2006). These transitions are excited by the GRB afterglow itself through indirect ultraviolet pumping (Prochaska et al. 2006; Vreeswijk et al. 2007) of gas in the interstellar medium (ISM) of the host galaxy. Altogether, the coincidence of (1) the absence of any higher redshift absorption systems in our spectrum, (2) the positive detection of finestructure Fe II transitions, and (3) the absence of intergalactic medium absorption at $\lambda>3500 \AA$ establishes $z=1.604$ as the redshift of GRB 071003.

It might seem unusual to have detected fine-structure Fe II transitions in such a late-time spectrum $(t \approx 24.3 \mathrm{hr})$. Because the lines are excited by the GRB afterglow, they will decay as the 
TABLE 7

Absorption Lines in the Afterglow Spectrum of GRB 071003

\begin{tabular}{|c|c|c|c|c|}
\hline $\begin{array}{c}\lambda \\
(\AA)\end{array}$ & $z$ & Transition & $\begin{array}{l}W^{\mathrm{a}} \\
(\AA)\end{array}$ & $\begin{array}{c}\sigma(W)^{\mathrm{b}} \\
(\AA)\end{array}$ \\
\hline $3549.69 \ldots \ldots$ & 0.37223 & $\mathrm{Fe}$ II $\lambda 2586$ & $<2.51$ & \\
\hline $3568.06 \ldots \ldots \ldots \ldots \ldots \ldots \ldots \ldots$ & 0.37223 & $\mathrm{Fe}$ ІІ $\lambda 2600$ & 2.33 & 0.59 \\
\hline $3837.72 \ldots$ & 0.37223 & Mg II $\lambda 2796$ & 2.48 & 0.20 \\
\hline $3847.65 \ldots$ & 0.37223 & Mg II $\lambda 2803$ & 2.14 & 0.19 \\
\hline 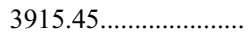 & 0.37223 & $\mathrm{Mg}$ I $\lambda 2852$ & 1.02 & 0.17 \\
\hline $4032.63 \ldots \ldots \ldots \ldots \ldots \ldots$ & 1.60435 & C IV $\lambda 1548$ & 0.22 & 0.06 \\
\hline 4039.88 ...................... & 1.60435 & $\mathrm{C}$ IV $\lambda 1550$ & $<0.28$ & $\ldots$ \\
\hline $4351.92 \ldots \ldots \ldots \ldots \ldots \ldots$ & 1.60435 & $\mathrm{Al}$ Іा $\lambda 1670$ & $<0.14$ & 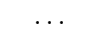 \\
\hline $5003.26 \ldots$. & 1.10019 & $\mathrm{Fe}$ пा $\lambda 2382$ & 0.20 & 0.05 \\
\hline $5276.54 \ldots \ldots$ & 1.60435 & Zn II $\lambda 2026$ & $<0.08$ & \\
\hline $5399.79 \ldots \ldots \ldots \ldots \ldots \ldots$ & 0.37223 & Ca II 23934 & 0.61 & 0.07 \\
\hline $5417.99 \ldots \ldots \ldots \ldots \ldots$ & 0.93740 & Mg II $\lambda 2796$ & 0.61 & 0.05 \\
\hline $5432.79^{c}$. & 1.10019 & $\mathrm{Fe}$ ІІ $\lambda 2586$ & 0.46 & 0.05 \\
\hline $5447.85 \ldots$ & 0.37223 & Ca II $\lambda 3969$ & 0.46 & 0.07 \\
\hline $5872.31 \ldots$ & 1.10019 & Mg II $\lambda 2796$ & 0.80 & 0.05 \\
\hline $5888.27 \ldots \ldots \ldots \ldots \ldots \ldots$ & 1.10019 & $\mathrm{Mg}$ II $\lambda 2803$ & 0.68 & 0.06 \\
\hline $6105.90 \ldots \ldots \ldots \ldots \ldots$ & 1.60435 & $\mathrm{Fe}$ II $\lambda 2344$ & $<0.17$ & .. \\
\hline 6206.91_................. & 1.60435 & $\mathrm{Fe}$ ІІ $\lambda 2382$ & 0.26 & 0.04 \\
\hline 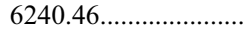 & 1.60435 & $\mathrm{Fe} \pi^{*} \lambda 2396 \mathrm{a}$ & 0.25 & 0.04 \\
\hline 6265.95 & 1.60435 & $\mathrm{Fe} \pi^{*} \lambda 2405$ & $<0.16$ & $\because$ \\
\hline $6282.68 \ldots \ldots \ldots \ldots \ldots \ldots \ldots$ & 1.60435 & $\mathrm{Fe} \mathrm{I}^{*} \lambda 2411 \mathrm{~b}$ & 0.18 & 0.03 \\
\hline $6284.57 \ldots \ldots \ldots \ldots \ldots \ldots . .$. & $\ldots$ & $\ldots$ & 0.72 & 0.12 \\
\hline $6734.47 \ldots \ldots \ldots \ldots \ldots \ldots$ & $\ldots$ & $\ldots$ & 0.97 & 0.15 \\
\hline $6737.28 \ldots \ldots \ldots \ldots \ldots \ldots$ & 1.60435 & Fe пा $\lambda 2586$ & 0.16 & 0.04 \\
\hline $6772.60 \ldots \ldots \ldots \ldots \ldots \ldots$ & 1.60435 & $\mathrm{Fe}$ пा $\lambda 2600$ & 0.27 & 0.05 \\
\hline $7301.58 \ldots \ldots \ldots \ldots \ldots \ldots$ & 1.60435 & Mg II $\lambda 2803$ & 0.17 & 0.05 \\
\hline $7430.06 \ldots \ldots \ldots \ldots \ldots \ldots$ & 1.60435 & $\mathrm{Mg}$ I $\lambda 2852$ & $<0.24$ & \\
\hline 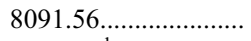 & $\ldots$ & $\ldots$ & 0.92 & 0.13 \\
\hline $8436.10^{\mathrm{d}} \ldots \ldots \ldots \ldots \ldots$ & $\ldots$ & $\ldots$ & 0.86 & 0.26 \\
\hline $8534.91^{\mathrm{d}} \ldots \ldots \ldots \ldots \ldots \ldots$ & $\ldots$ & $\ldots$ & 0.72 & 0.17 \\
\hline $8599.02^{\mathrm{d}} \ldots \ldots \ldots \ldots \ldots \ldots$ & $\ldots$ & $\ldots$ & 1.34 & 0.17 \\
\hline
\end{tabular}

${ }^{a}$ Equivalent widths are rest-frame values and assume the redshift given in the second column

${ }^{\mathrm{b}}$ Limits are $2 \sigma$ statistical values.

${ }^{c}$ Blended with $\mathrm{Mg}$ II 22803 at $z=0.937$.

d These features may be residuals from sky subtraction.

afterglow fades on hour-long timescales (Dessauges-Zavadsky et al. 2006; Vreeswijk et al. 2007; D'Elia et al. 2008). The presence of fine-structure transitions in our spectrum, however, is consistent with the late-time rebrightening of GRB 071003 provided that the gas lies within a few kiloparsecs of the GRB. In Figure 9 we present a velocity plot of strong resonance-line transitions for $z=z_{\mathrm{GRB}}$. We report the positive detections of $\mathrm{C}$ IV $\lambda 1548, \mathrm{Fe}$ II $\lambda \lambda 2382,2586,2600$, and $\mathrm{Mg}$ II $\lambda 2803$, and we note probable but statistically insignificant absorption at $\mathrm{Al}$ II $\lambda 1670$ and $\mathrm{Mg}$ II $\lambda 2796$. The rest-frame equivalent widths are among the lowest ever recorded for the ISM surrounding long-duration GRBs. The equivalent width of $\mathrm{Mg}$ II, for example, is fully an order of magnitude below the general population (Cenko et al. 2008), with the sole exception of GRB 070125, and the equivalent width for the $\mathrm{C}$ iv gas $\left(W_{1548}=0.22 \pm 0.06 \AA\right)$ represents the lowest measurement to date (Prochaska et al. 2008).

\section{RESULTS AND MODELING}

\subsection{Light Curve: General Observations}

The multicolor photometric evolution of the GRB 071003 OA is shown in Figure 10, fitted by our preferred model (described later). Visual inspection of the light curves reveals what appear to be three distinct components: an overall power-law decline that has already set in by the very first measurement at $42 \mathrm{~s}$, a small "bump" feature at $\sim 120-600 \mathrm{~s}$, and then a dramatic, but unfortunately not well sampled, rebrightening starting around $3000 \mathrm{~s}$ that dominates the remainder of the evolution.

The bump feature appears to be additive only: fitting a single power law to measure the decay index $\left(t^{-\alpha}\right)$ for the clear-band data both before this period and after it, the power-law indices $(\alpha=$ 1.47 and 1.49 , respectively) are fully consistent with each other and with the overall decay index over both periods $(\alpha=1.48)$.

The rebrightening is more difficult to characterize. We have no observations between the Keck I HIRES guider point at $t \approx 2.6 \mathrm{hr}$ and our observations the second night; moreover, the points reported in the GCNs are highly discrepant. An optical $R$-band limit is reported at $t \approx 4 \mathrm{hr}$ by Shih et al. (2007), which seems to contradict the rebrightening trend suggested by the AEOS data and guider point. It is unlikely that the OA would show such a dramatic drop ( $>3 \mathrm{mag}$ ) in a short time interval at such late times, so we suspect that the OA might be heavily contaminated by the bright nearby star and was not resolved in the Lulin $1 \mathrm{~m}$ telescope images of Shih et al. (2007). On the other hand, the $U$-band detection at $t \approx 7.5 \mathrm{hr}$ reported by Misra et al. (2007) supports a rebrightening but is several magnitudes above the extrapolated light curve at this time, seemingly far too bright to be consistent with our observations. Calibration and the contamination from the bright star are the likely causes of the discrepancy.

\subsection{Optical to Gamma-Ray and X-Ray Comparison}

The BAT and XRT light curves we derive for GRB 071003 are also shown in Figure 10. Unfortunately, because Swift was still in the process of returning to normal operations after its gyro failure (Gehrels 2007), automatic slewing to GRB 071003 was disabled at the time when the GRB was detected. As a result, there were no prompt XRT observations for GRB 071003, leaving a long gap in the gamma-ray/X-ray light curve at $t=200-20,000 \mathrm{~s}$. In particular, there are no X-ray observations until approximately the peak of the rebrightening in the optical band. Nevertheless, direct comparison of the data available reveals three relevant facts.

First, there is no obvious optical prompt counterpart to the last spike of the gamma-ray light curve. However, this spike is nearly contemporaneous with the much more slowly rising optical bump feature; we return to this possible connection in our later modeling ( $(4.5)$.

Second, at late times the X-ray light curve declines as a power law with decay index consistent with that observed in the optical. A simple power law fits the data well, with a best-fit decay index of $\alpha_{\mathrm{X}}=1.68 \pm 0.04$. In addition, the late-time OA behavior (after $t \approx 5 \times 10^{4} \mathrm{~s}$ ) is consistent with a single power-law decay with an index of $\alpha_{O}=1.72 \pm 0.07$, fully consistent with this value. As we note later, an extrapolation of the X-ray spectral index is also consistent with the optical observations, suggesting that at late times there is no need for an additional X-ray contribution (such as inverse Compton) or large amounts of host galaxy extinction.

Finally, while the gamma rays are scaled arbitrarily in Figure 10, we note that if we extrapolate the gamma-ray spectrum into the X-rays to compare the BAT and XRT light curves, the evolution between the end of the prompt emission and the start of the XRT observations is nearly consistent with a simple extension of the late-time XRT power law back to earlier times, without a need for a rebrightening or break. However, Swift has shown previously (Nousek et al. 2006) that early-time X-ray light curves can conceal a wide variety of complex features, so we will not speculate further as to whether or not this was actually the case. 

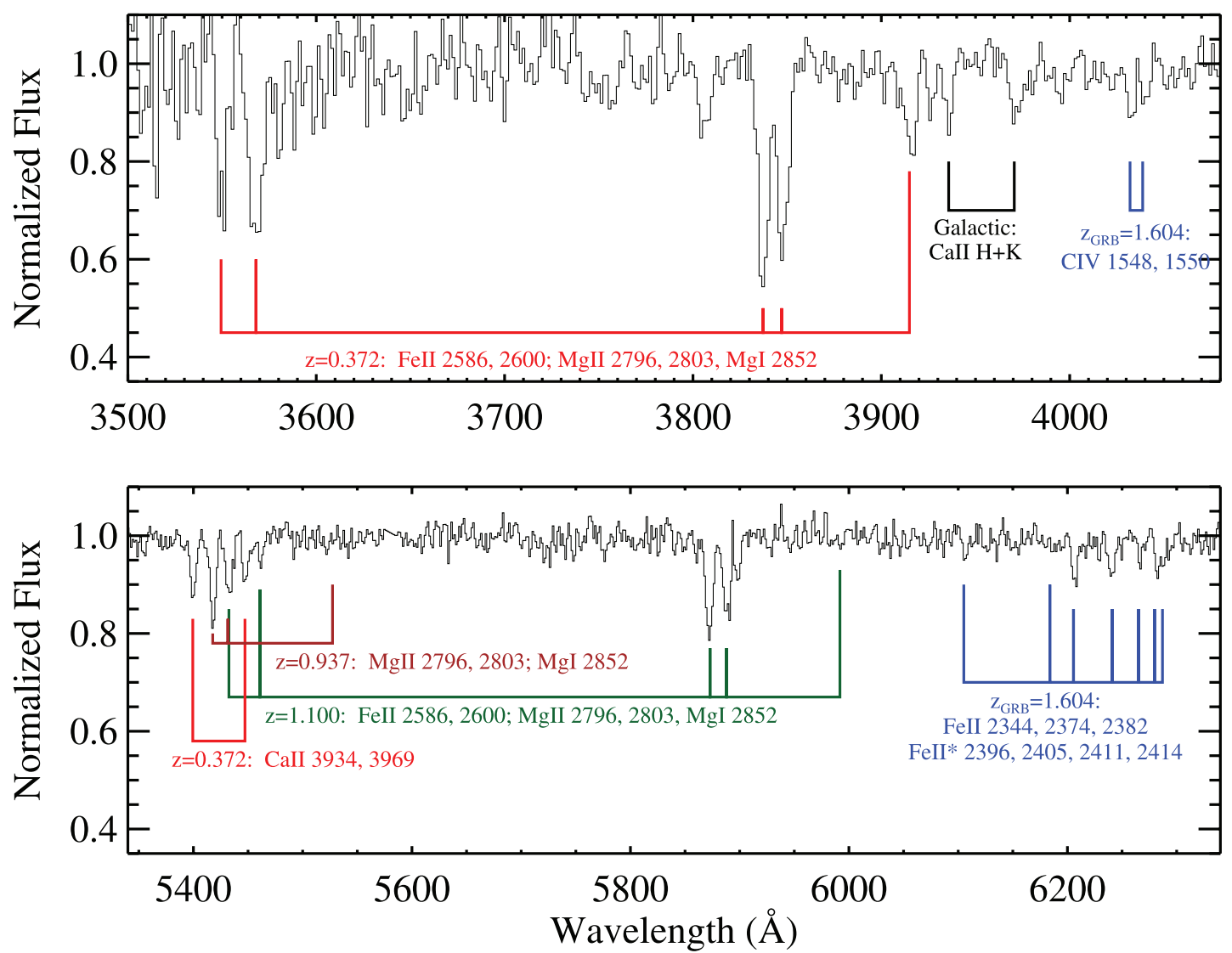

FIG. 8.-Portions of the normalized Keck LRIS spectrum of the GRB 071003 afterglow. We mark the positions of several metal absorption line features from four distinct extragalactic systems, including a series of $\mathrm{Fe}$ II and $\mathrm{Fe}_{\mathrm{II}}^{*}$ transitions associated with the host galaxy of GRB $071003\left(z_{\mathrm{GRB}}=1.604\right)$. Note that the Ca II doublet marked as Galactic may be due to the very bright Galactic star offset by $6.5^{\prime \prime}$ from GRB 071003 as opposed to the Galactic ISM.

\subsection{Detailed Optical Modeling}

The procedure used to model the optical light curve is generally the same as that employed by Perley et al. (2008) but further generalized. For our fit model, we employ an unbroken powerlaw decay (component 0) plus two Beuermann et al. (1999) functions (broken power-law pairs, components 1 and 2) but allow for different values of the functional parameters for each filter and component. The functional form is

$$
\begin{aligned}
F_{\nu}= & F_{0, \nu}\left(t-d t_{0}\right)^{-\alpha_{0}} \\
& +F_{1, \nu}\left[0.5\left(\frac{t-d t_{1}}{t_{p 1}}\right)^{-s_{1} \alpha_{1, b}}+0.5\left(\frac{t-d t_{1}}{t_{p 1}}\right)^{-s_{1} \alpha_{1, a}}\right]^{-1 / s_{1}} \\
& +F_{2, \nu}\left[0.5\left(\frac{t-d t_{2}}{t_{p 2}}\right)^{-s_{2} \alpha_{2, b}}+0.5\left(\frac{t-d t_{2}}{t_{p 2}}\right)^{-s_{2} \alpha_{2, a}}\right]^{-1 / s_{2}},
\end{aligned}
$$

where for component $0, \alpha_{0}$ is the power-law decay index and $d t_{0}$ is an adjustment to the Swift BAT trigger time. For component 1, $\alpha_{1, b}$ and $\alpha_{1, a}$ are the power-law decay indices for the rising and declining components, respectively, $d t_{1}$ is an adjustment to the Swift BAT trigger time, $t_{p 1}$ is the time of the peak flux, and $s_{1}$ is the sharpness parameter. Component 2 has a similar function as component 1 .

Fitting this function with no constraints generates unrealistic results because of nonuniform sampling in different filters. However, we can make the following physically motivated assump- tions to tie specific parameters and produce more physically meaningful results:

1. We assume that the temporal decay index at any given time is independent of the filter, as is implicit in the notation ( $\alpha$ does not depend on $\nu$ ). This means that the color of a component cannot change except while the light curve of that component is breaking.

2. Most importantly, we assume that differences between the spectra of the various model components can be described by changes in the power-law index of the intrinsic spectrum, modified by an arbitrary, but fixed, extinction law. Mathematically, this constraint is expressed as $F_{i, \nu}=\nu^{\Delta \beta_{i j}} F_{j, \nu}$. Physically, this assumption requires that external effects such as extinction, which might cause the spectrum of any component to deviate from a power law, affect all components equally and are not time dependent. The extinction law itself (as well as the absolute underlying index of any specific component) is fully general and can be fitted according to various models later.

3. In addition, we assume that the rising segments of each component are also power laws, but not necessarily the same power laws as the falling segment, to allow for chromatic breaks. This imposes the following condition: $\left(t_{p}, x\right) /\left(t_{p}, y\right)=\left(\nu_{y} / \nu_{x}\right)^{\Delta \beta_{b a} / \Delta \alpha_{b a}}$. Here $b$ and $a$ refer to "before" and "after" the break of a specific component $(0,1$, or 2$)$, where the component index is omitted for clarity, and $x$ and $y$ refer to two different filters.

Fitting is performed, under these assumptions, using the IDL package MPFIT. ${ }^{27}$

${ }^{27}$ See http://cow.physics.wisc.edu/ craigm/idl/idl.html. 


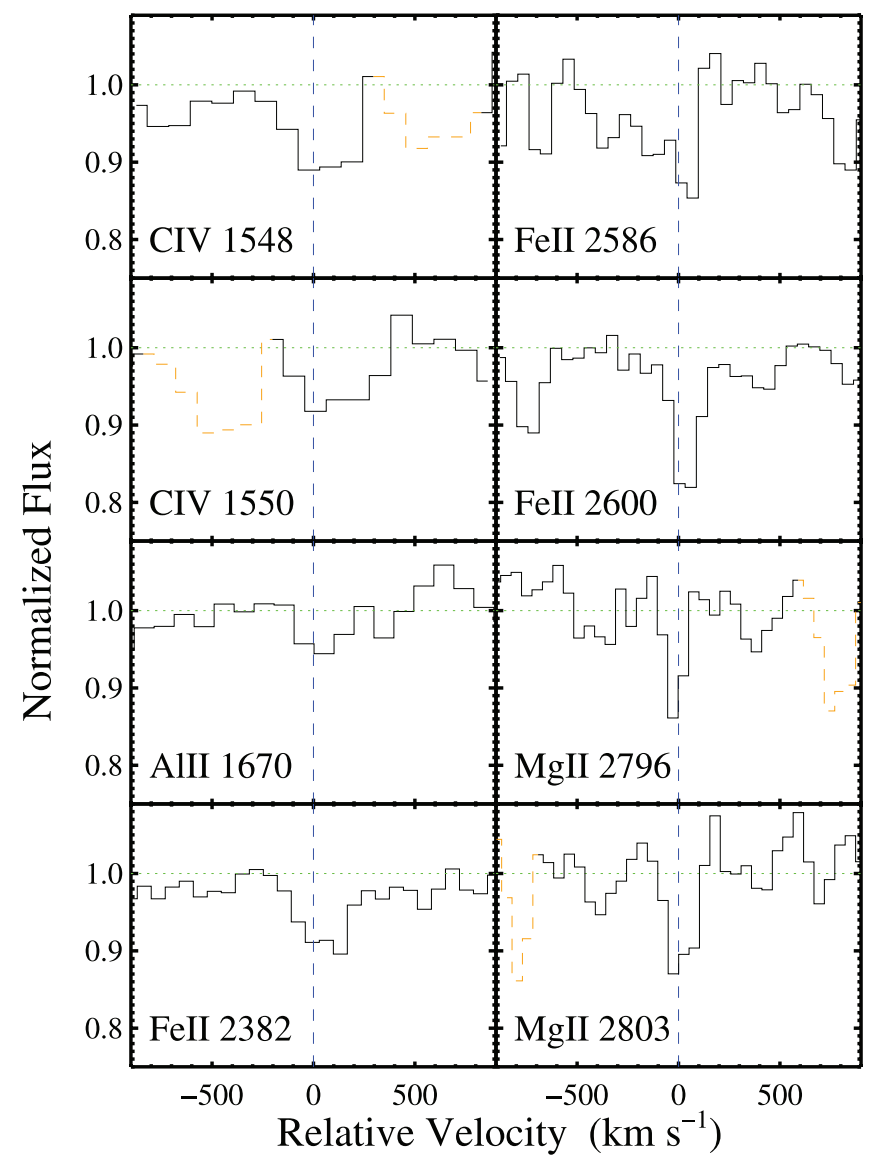

FIG. 9.- Velocity plot of strong, resonance-line transitions for gas associated with GRB $071003\left(z_{\mathrm{GRB}}=1.60435\right)$. These lines are very weak (note the ordinate scale) with rest-frame equivalent widths of 100-200 m $\AA$ ( Table 7). Indeed, the C IV absorption is the weakest yet reported for a GRB afterglow (Prochaska et al. 2008).

The assumptions involved in these constraints are, of course, oversimplifications for the full array of models that might be considered. In particular, this model allows only one break per component, but with an evolving synchrotron light curve plus a jet we may expect as many as three. However, it has the advantage of being simple and generates a single physically motivated parameter quantifying color change over each component.

We perform a variety of fits under varying combinations of assumptions. Some of the possibilities we considered include the following:

1. Forcing the bump (component 1) to have the same color as the uniform decay (component 0 ), or allowing it to be a different color overall.

2. Forcing the bump itself to be achromatic over its evolution, or allowing it to contain a chromatic break.

3 . Forcing the late rebrightening (component 2) to have the same color as the uniform decay, or allowing it to have a different color.

4. Fixing $d t_{0}$ for the early steep decay to be zero (the BAT trigger time), or allowing it to be free to vary.

5 . Fixing $d t_{1}$ for the bump component to be zero, to be equal to the beginning of the prompt-emission pulse that is nearly contemporaneous with it, or allowing it to be free to vary.

6. Fixing $d t_{2}$ for the late rebrightening to be zero, or allowing it to be free to vary.

The results under various combinations of these assumptions are presented in Tables 8 and 9. We discuss the implications of these results in the remainder of the paper.

\subsection{Color Change}

Detection of a GRB afterglow in filtered observations during prompt emission, as was the case here, is rare. The situation is even more intriguing since our multicolor prompt OA observations show an apparent bump feature (component 1) that is nearly contemporaneous with a rebrightening pulse in the gamma-ray light curve. Therefore, it is of great interest to attempt to measure the color of component 1. By the same token, we have good spectral coverage of the afterglow both during the primary normal decay and during the fading of the dramatic late rebrightening, and any color difference may shed light on the origin of these features.

We tested for color differences in three places: between component 0 (rapid decay) and component 1 (bump), between component 0 and component 2 (rebrightening), and over the break of component 1 itself (since the rising spectral index may differ from the falling spectral index). In all cases we find evidence for color variation, although in each case only at the $\sim 2 \sigma$ level. The fading component of the bump is redder than the fading component of the uniform decay by $\Delta \beta=0.75 \pm 0.34$, the bump feature is chromatic with a shift from the rising to falling component of $\Delta \beta=1.11 \pm 0.47$, and the rebrightening (for which we only have color information during the fading component) is also redder, by $\Delta \beta=0.84 \pm 0.31$.

One must be somewhat cautious in interpreting these results: since different filters sample the data differently, systematic errors that affect only one portion of the light curve can masquerade as color change. Data reduction for GRB 071003 was also challenging due to the presence of the nearby bright star, as detailed in $\S 3$. In addition, we note that the degree of spectral index shifts noted is dependent on the model. In spite of these considerations, however, we feel that our conclusion of color change is reasonably secure in each case.

\subsection{Energy Injection Times}

It is often unclear what time is most appropriate to use as $t_{0}$ when fitting a power law to a GRB afterglow. Thanks to the extremely early-time clear-band data, it is possible to fit $t_{0}$ and constrain this within a few seconds in the case of GRB 071003. This fit, notably, gives a $t_{0}$ of exactly the trigger time $\left(d t_{0}=\right.$ $-0.01 \pm 3.01 \mathrm{~s}$ ). The gamma-ray light curve (Fig. 1) fluence is strongly dominated by the initial pulse, which rises sharply and peaks within a few seconds, so this is not necessarily surprising.

Some authors (Blake et al. 2005; Vestrand et al. 2005, 2006; Yost et al. 2007) have presented evidence of an optical component rising coincident with the prompt emission, although significantly longer lasting. We can analyze whether the bump component observed in GRB 071003 may be such a feature by determining whether or not it can be fitted with a pulse that rises abruptly, contemporaneous with the prompt emission. While our power-law model is somewhat simplified and the sampling of the rise is extremely poor, we find that it generally does not: the best-fit $t_{0}$ is intermediate between the trigger time and the time of the promptemission spike $(\sim 125 \mathrm{~s})$ at $d t_{1}=60 \pm 20 \mathrm{~s}$. This is a modelindependent result, although it rests mostly on one data point: the initial $V$-band measurement, representing an integration from 97 to $117 \mathrm{~s}$ after the BAT trigger ( $\sim 18 \mathrm{~s}$ before the rise of the promptemission spike), lies 0.14 mag above a simple power-law extrapolation from regions of the data excluding the bump, compared to a photometric error of only $0.03 \mathrm{mag}$. While it is possible to envision scenarios where a relatively slow optical rise might follow a gamma-ray pulse (any broadband feature with hard-tosoft evolution, or perhaps a late internal shock that later collides 


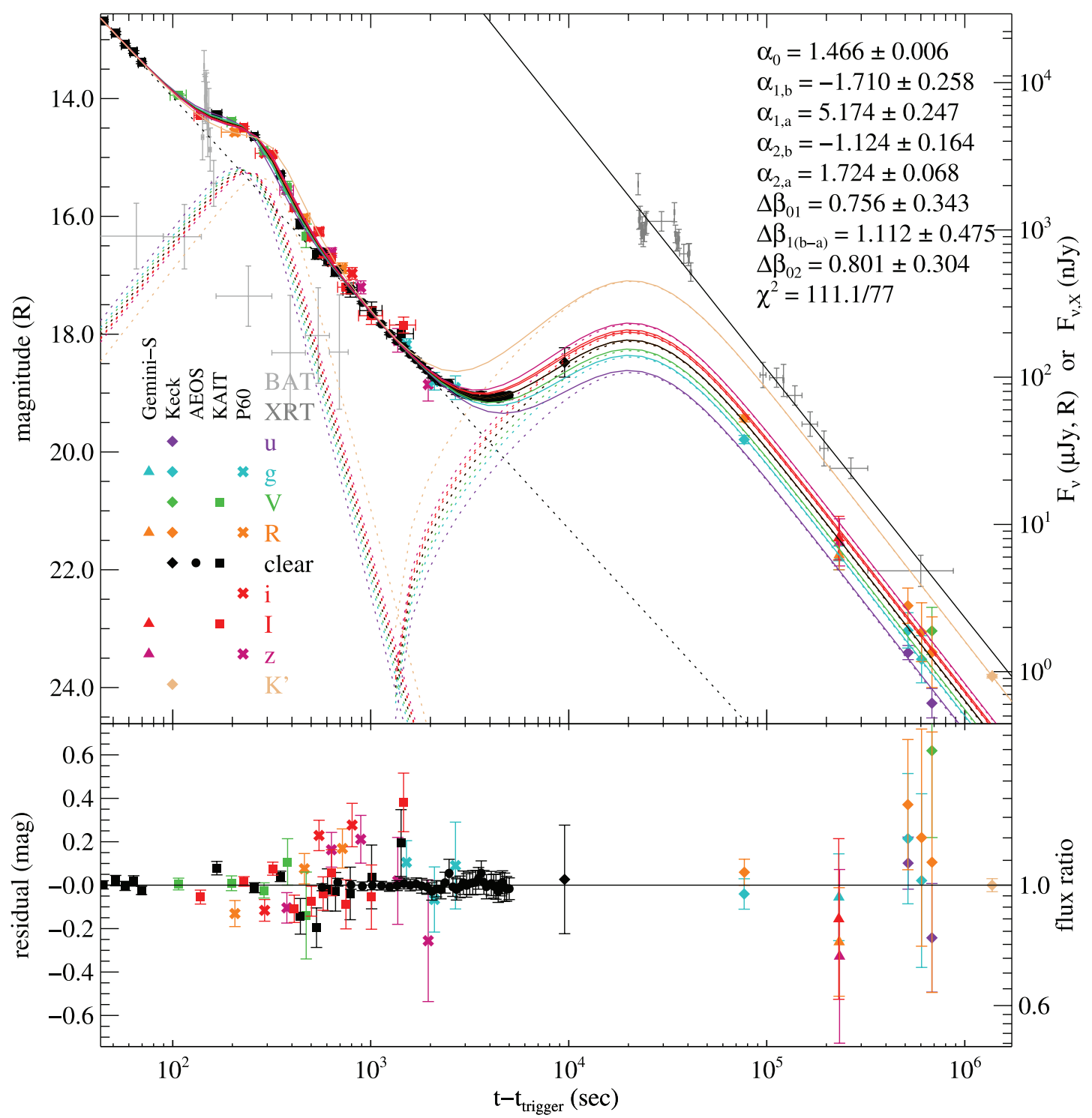

FIG. 10.- Multicolor, early- through late-time light curves of the OA of GRB 071003. The magnitudes are offset according to their early-time colors, showing the color evolution between early and late times. Overplotted colored lines indicate the best-fit three-component, color evolution model described in the text; the dashed lines represent the individual components that compose this model (a uniform power-law decay, a chromatic early-time bump, and a monochromatic late-time rebrightening). The X-ray and gamma-ray afterglows are also overplotted for comparison. The gamma-ray light curve is scaled arbitrarily; if scaled based on the likely gamma-ray-to-X-ray spectral index, it would fall on or near the extrapolation of the X-ray light curve back to early times.

with and energizes the external shock), no model to our knowledge can explain why an optical flare would precede a gammaray pulse, so we take this as evidence that the two features are physically unconnected.

While our sampling around the rise and peak of the late-time rebrightening is poor (and dominated by the difficult-to-calibrate
AEOS and HIRES guider images), we can also attempt to fit the $t_{0}$ for the rebrightening component. This is significantly different from $t=0$, with a best-fit initial time of $d t_{2}=1245 \pm 311 \mathrm{~s}$. (This is well short of its peak time of approximately $20 \mathrm{ks}$, so the effect on the light curve is minor.) No promptlike fluctuations or other features are observed in the light curve in this region.

TABLE 8

Optical Light-Curve Fits: Color Change

\begin{tabular}{|c|c|c|c|c|c|}
\hline Model Description & $\Delta \beta_{0-1}$ & $\Delta \beta_{1}(b-a)$ & $\Delta \beta_{0-2}$ & $\beta_{2}$ & $\chi^{2} / \nu$ \\
\hline 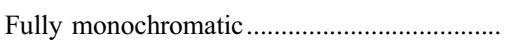 & 0 & 0 & 0 & $0.72 \pm 0.10$ & $125.765 / 81$ \\
\hline 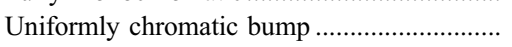 & $0.22 \pm 0.27$ & 0 & 0 & $0.68 \pm 0.10$ & $125.100 / 80$ \\
\hline 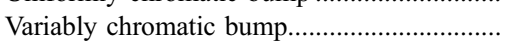 & $0.66 \pm 0.33$ & $1.05 \pm 0.47$ & 0 & $0.70 \pm 0.10$ & $120.339 / 79$ \\
\hline 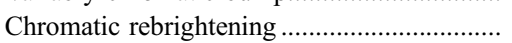 & 0 & 0 & $0.77 \pm 0.31$ & $1.26 \pm 0.11$ & $120.040 / 80$ \\
\hline Chromatic bump+rebrightening ...................... & $0.75 \pm 0.33$ & $1.09 \pm 0.47$ & $0.84 \pm 0.31$ & $1.26 \pm 0.11$ & $113.713 / 78$ \\
\hline
\end{tabular}

NoтES.-Summary of relevant parameters and $\chi^{2}$ for models allowing or disallowing color transitions and chromatic breaks between the various components. Values without uncertainties are fixed. Component 0 is the fast-decay component, component 1 is the bump, and component 2 is the late rebrightening. The absolute late-time spectral index $\beta_{2}$ is not a model parameter but is fitted externally after completion of the fit. 
TABLE 9

Optical Light-Curve Fits: $t_{0}$

\begin{tabular}{|c|c|c|c|c|}
\hline Model Description & $\begin{array}{r}d t_{0} \\
(\mathrm{~s})\end{array}$ & $\begin{array}{l}d t_{1} \\
\text { (s) }\end{array}$ & $\begin{array}{l}d t_{2} \\
(\mathrm{~s})\end{array}$ & $\chi^{2} / \nu$ \\
\hline 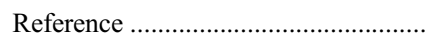 & 0 & 0 & 0 & $113.713 / 78$ \\
\hline Decay . . & $-0.01 \pm 3.01$ & 0 & 0 & $113.713 / 77$ \\
\hline Bump & 0 & $60.5 \pm 20.4$ & 0 & $112.700 / 77$ \\
\hline 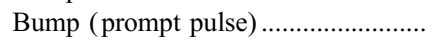 & 0 & 125.0 & 0 & $115.118 / 78$ \\
\hline 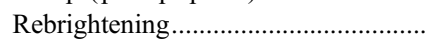 & 0 & 0 & $1245 \pm 311$ & $111.149 / 77$ \\
\hline
\end{tabular}

Notes.-Summary of relevant parameters and $\chi^{2}$ for models using a $t_{0}$ different from the trigger time. In all cases, the favored color-change model (chromatic bump and rebrightening) was used. Values without uncertainties are fixed. Component 0 is the fast-decay component, component 1 is the bump, and component 2 is the late rebrightening.

\subsection{Radio Modeling}

GRB 071003 is rare among Swift bursts for having a bright radio afterglow. We were able to successfully detect the afterglow at two frequencies and several epochs spanning $\sim 2-20$ days after the burst, including observations nearly contemporaneous with our optical data. The data are plotted in Figure 11.

This GRB is not far off the Galactic plane, and the radio observations are affected by scintillation. Following Walker (1998, 2001), the afterglow is in the strong scattering regime for both $\mathrm{X}$ and $\mathrm{C}$ bands. An approximate modulation index (which estimates the fractional rms variation) is 0.4 in the $\mathrm{C}$ band and 0.6 in the $\mathrm{X}$ band, over a refractive timescale of $\sim 0.5$ days in the $\mathrm{X}$ band and 2 days in the $\mathrm{C}$ band. This is longer than any integration (so the error is not reduced by integration time) but shorter than the interval between exposures (so errors are uncorrelated).

Radio data were fitted using both an unbroken power-law model and a singly broken power-law model. We attempted the fit both before including uncertainties due to scintillation and with an additional $40 \%$ flux error added to all C-band points and $60 \%$ error added to all X-band points.

Without the additional flux errors, the unbroken model is a poor fit, with $\chi^{2} / \nu=15.32 / 6$. A single, monochromatic break improves the fit dramatically $\left(\chi^{2} / \nu=2.45 / 4\right)$. This improvement is significant at $97.4 \%$ confidence. A monochromatic radio break of this nature is very difficult to explain physically. However,

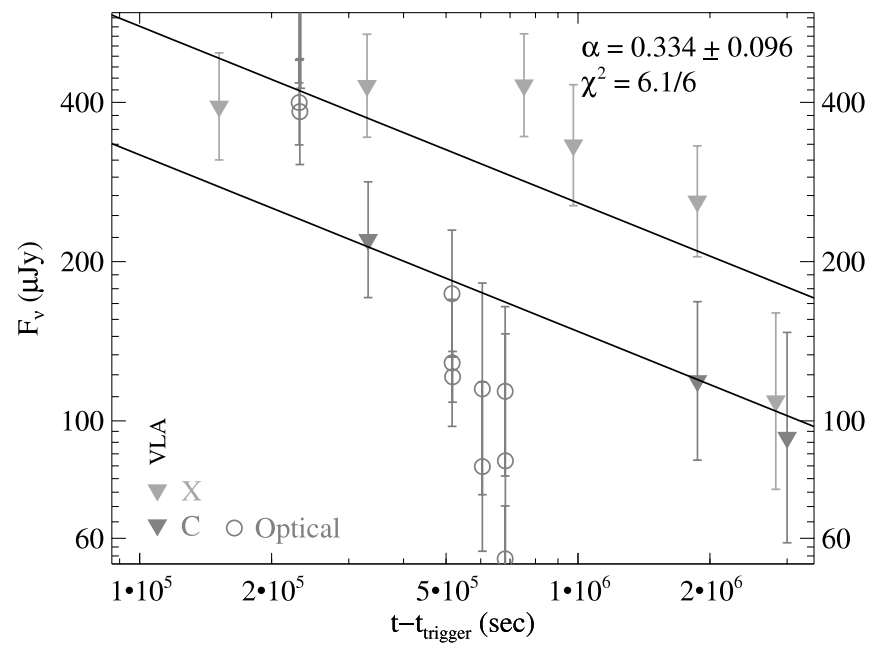

FIG. 11. - VLA radio light curve fitted to an unbroken power law. The uncertainties in the measurements have been increased compared to their statistical values to take into account the effect of interstellar scintillation. Some contemporaneous late-time optical points (scaled arbitrarily) are shown for comparison. [See the electronic edition of the Journal for a color version of this figure.] with scintillation flux errors folded into the light curve, we find that a simple power law is a more than adequate fit to the data $\left(\chi^{2} / \nu=1.4 / 6\right)$, which may suggest that we have overestimated the degree of modulation somewhat. (This is to be expected: the modulation index calculated is an upper limit as it strictly applies only for a point source. The afterglow has a physical size, which quenches the scintillation modulation somewhat.) Therefore, as a final modification, we scaled down this additional error until the final $\chi^{2} / \nu \approx 1$. Properties of the temporal fits are given in Table 10 .

The uncertainty due to scintillation is in any event too large to allow any firm conclusions about the light curve. However, since only refractive scintillation is expected to be significant, the refractive timescale is much longer than the several-hour timescale of individual observations, and the C-band observations were in all cases taken immediately after the X-band observations, we do consider the measurement of the radio spectral index $\left(\beta_{R}=\right.$ $-1.15 \pm 0.42)$ to be trustworthy regardless of any scintillation uncertainty.

\subsection{Spectral Energy Distribution and Extragalactic Extinction}

If our modeling assumptions are accurate (or nearly so), we can use our model to calculate the SED at any time using a combination of all the data available, rather than restricting the measurement to a small subset of the photometry and filters, even if the data were acquired at very different times during the evolution of the GRB OA and the color were not constant.

We calculate the SED at two epochs. First, we calculate the SED at $t=2.67$ days after the burst, the time of our four-color Gemini-South observations. In calculating this SED, we perform a slightly modified light-curve fit: we do not perform any filter transformations (e.g., to convert $r$ to $R$ ), but we fix all non-SED parameters to that derived from the light-curve analysis. In addition, we add in quadrature a calibration uncertainty equal to $5 \%$ in all filters, with a few exceptions. For $z$, we use a $15 \%$ uncertainty. For $u$, we use a $30 \%$ uncertainty, for reasons described earlier. Finally, for $K^{\prime}$, we use a large extra uncertainty of $50 \%$ due to the possibility of a temporal break sometime between our last optical observations and the $\mathrm{AO}$ observations. (However, if such a break is absent, then the $K^{\prime}$ observation is much more precise than is given on the plots.) Unfiltered observations are not used. We also calculate an early-time SED during the "normal" power-law decay at $t=1000 \mathrm{~s}$, using a fit excluding late-time measurements and measurements during the (possibly chromatic) bump. Addition of uncertainties is as for the late-time SED.

The resulting SEDs are plotted in Figures 12 and 13. After removing the effects of Galactic extinction (but not yet considering non-Galactic extinction), both SEDs are a reasonable fit to 
TABLE 10

Radio Modeling of GRB 071003

\begin{tabular}{|c|c|c|c|}
\hline Parameter & Value (Broken Power Law) & Value (Unbroken) & Value (Unbroken w/Scintillation) ${ }^{\mathrm{a}}$ \\
\hline 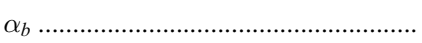 & $-0.11 \pm 0.21$ & $0.27 \pm 0.06$ & $0.34 \pm 0.10$ \\
\hline . n................... & $0.81 \pm 0.25$ & $\ldots$ & $\ldots$ \\
\hline 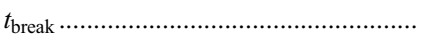 & $8.51 \pm 3.78$ & $\ldots$ & $\ldots$ \\
\hline$\beta$ & $-1.11 \pm 0.34$ & $-1.15 \pm 0.44$ & $-1.15 \pm 0.42$ \\
\hline 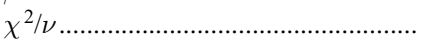 & $2.45 / 4$ & $15.32 / 6$ & $6.07 / 6$ \\
\hline
\end{tabular}

NoTES.-Best-fit parameters of a fit to the radio afterglow of GRB 071003 using a Beuermann et al. (1999) broken power-law model vs. an unbroken power-law model. The improvement for the broken power-law fit is significant given the flux uncertainties, but due to interstellar scintillation may be coincidental. If a small amount of interstellar scintillation uncertainty is added in quadrature, an unbroken power-law fit is reasonable.

${ }^{a}$ In this model, we added a $15 \%$ error to all X-band points and a $22 \%$ error to all C-band points.

a power law, providing a general confirmation of our assumptions, as well as indicating that the host or intervening galaxies do not impose a great deal of frequency-dependent extinction. In support of our analysis from the light-curve modeling, the spectral indices appear to differ from early to late times: $\beta_{1000 \mathrm{~s}}=0.62 \pm 0.33$, while $\beta_{2.67 \text { days }}=1.25 \pm 0.09$. (These values are direct fits to the data and do not include the effects of the small amount of extragalactic extinction we do believe to be present, which we discuss shortly.)

Unfortunately, there were no early-time observations outside the optical band, since Swift was unable to slew rapidly. However, this GRB was observed nearly simultaneously in X-rays, optical, and radio during the declining phase of the late rebrightening. Therefore, it is possible to calculate a coeval late-time spectrum at all wavelengths simultaneously. The values at 2.67 days (the same as the first optical-only SED, above, which is also contemporaneous with XRT observations and within about half a day of the first VLA observation) are given in Table 11 and plotted in Figure 14.

Even without considering host galaxy extinction, the optical and X-ray observations are nearly consistent with a common spectral index: $\beta_{O}=1.25 \pm 0.09, \beta_{\mathrm{X}}=1.14 \pm 0.12$, and $\beta_{\mathrm{OX}}=$ $0.90 \pm 0.03$. This consistency, plus the fact that the optical and $\mathrm{X}$-ray temporal decays are identical $\left(\alpha_{O}=1.72 \pm 0.31, \alpha_{\mathrm{X}}=\right.$ $1.68 \pm 0.05$ ), argues that both X-ray and optical are in the same synchrotron regime and the spectrum across this range is a sim-

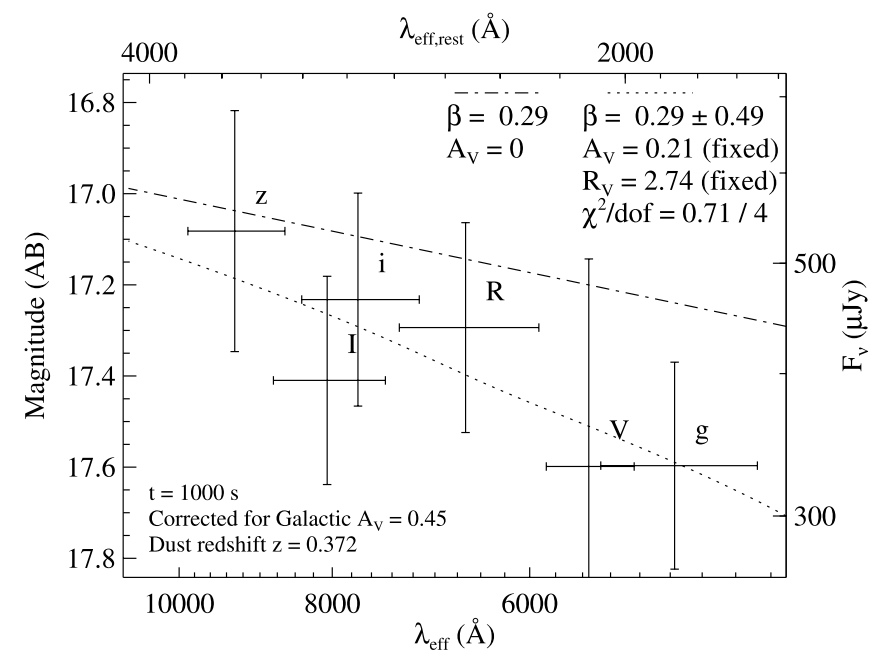

FIG. 12.- Optical SED of the GRB 071003 OA at $1000 \mathrm{~s}$ after the burst, fitted using the extinction constraints derived using the late-time SED. The intrinsic (preextinction) model spectrum is also shown. [See the electronic edition of the Journal for a color version of this figure.] ple power law. We assume this throughout the remainder of the analysis.

The deviations in the observed spectral index suggest the presence of a small amount of extragalactic extinction. Because of the presence of numerous absorbers and the unusually weak nature of the highest redshift absorption system, however, the appropriate assumptions for modeling the extinction contribution are not clear. Although $\mathrm{Mg}$ II is not an exact tracer of the presence of dust, the extremely weak line absorption at the likely host galaxy redshift of $z=1.604$ suggests that the dust column at that redshift is nearly negligible. Among the remaining absorbers, the $\mathrm{Mg}$ II system at $z=0.372$ is by far the strongest (by a factor of $\sim 3$ in equivalent width compared to the next strongest system at $z=1.10$ ) and is likely to be the dominant contributor to any observed dust absorption. However, this is partially offset by the fact that dust at higher redshift is much more opaque (since the observed optical frequencies are in the rest-frame UV at $z>1$ ), so for the moment we remain agnostic as to the actual redshift of the absorbing dust.

We fit the optical spectrum simultaneously with the normalized X-ray flux of $F_{1 \mathrm{kev}}=0.036 \pm 0.004 \mu \mathrm{Jy}$ at 2.67 days. This value has already been corrected for photoelectric absorption $(\S 2.1)$, and $\mathrm{X}$-ray absorption is not considered in the fit, allowing the gas-todust ratio to be independent of the amount of extinction, $A_{V}$.

Four different extinction models were tested. In addition to a control fit with no extinction, we fit for Milky Way-like, Small

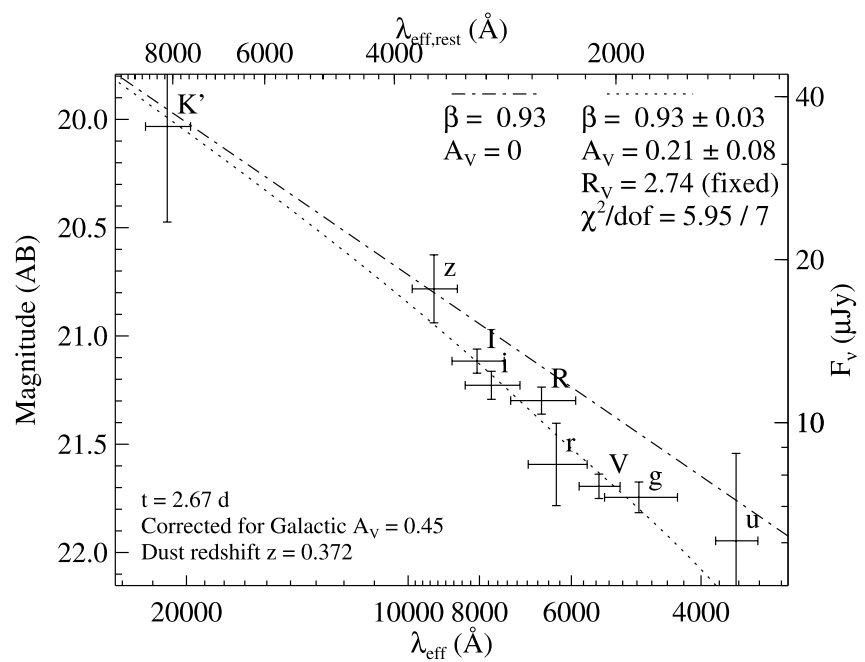

FIG. 13.- Same as Fig. 12, but for $t=2.67$ days after the burst. The data (plus an X-ray normalization, not shown) have been fitted with an SMC-like extinction law, with the best-fit line overplotted. The intrinsic (preextinction) model spectrum is also shown.[See the electronic edition of the Journal for a color version of this figure.] 
TABLE 11

Model FluXes at $t=2.67$ days

\begin{tabular}{|c|c|c|c|}
\hline Band/Filter & $\begin{array}{c}E \\
(\mathrm{eV})\end{array}$ & $\begin{array}{l}\text { Flux } \\
(\mu \mathrm{Jy})\end{array}$ & $\begin{array}{c}\text { Uncertainty } \\
(\mu \mathrm{Jy})\end{array}$ \\
\hline $\mathrm{X}$-ray ............ & 1000 & 0.036 & 0.006 \\
\hline u.............. & 3.46 & 3.17 & 1.42 \\
\hline$g$. & 2.55 & 4.47 & 0.30 \\
\hline$V$ & 2.25 & 5.07 & 0.27 \\
\hline 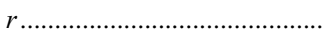 & 1.97 & 5.97 & 1.14 \\
\hline ................. & 1.88 & 8.01 & 0.47 \\
\hline 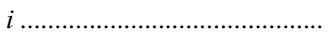 & 1.61 & 9.16 & 0.56 \\
\hline$I$ & 1.54 & 10.34 & 0.54 \\
\hline ................ & 1.34 & 14.74 & 2.29 \\
\hline .................. & 0.584 & 33.59 & 16.8 \\
\hline $\mathrm{X}$ & $3.5 \mathrm{E}-5$ & 414.6 & 91.8 \\
\hline 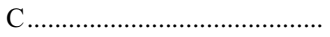 & $2.0 \mathrm{E}-5$ & 256.1 & 73.9 \\
\hline
\end{tabular}

Notes.-Fluxes of the afterglow interpolated to $t=2.67$ days after the BAT trigger using all available X-ray, optical, and radio data. Galactic extinction $[E(B-$ $V)=0.148 \mathrm{mag}]$ is not accounted for; however, the X-ray flux is corrected for photoelectric absorption.

Magellanic Cloud (SMC)-like, and Large Magellanic Cloud (LMC)-like extinction using the parameterization of the Fitzpatrick \& Massa (1990) ("FM") model and a model for extinction in starburst galaxies parameterized by Calzetti et al. (2000). In all cases the standard average value of the ratio of total to selective extinction $R_{V}$ in the reference galaxy in question was used. (Fits with varying $R_{V}$ were attempted, but lacking infrared or ultraviolet measurements we were unable to constrain this parameter.) We performed separate fits assuming dust at $z=0.372,1.100$, and 1.604 .

Results are given in Table 12. We find significant evidence ( $F$-test: $96 \%$ confidence) for a small amount $\left(A_{V}=0.1-0.3 \mathrm{mag}\right.$, depending on the model) of extinction along the line of sight. We cannot strongly constrain its nature; all four extinction laws, at each of the three possible redshifts, give reasonable fits to the observations. The intrinsic (preextinction) spectral slope $\beta$ is strongly

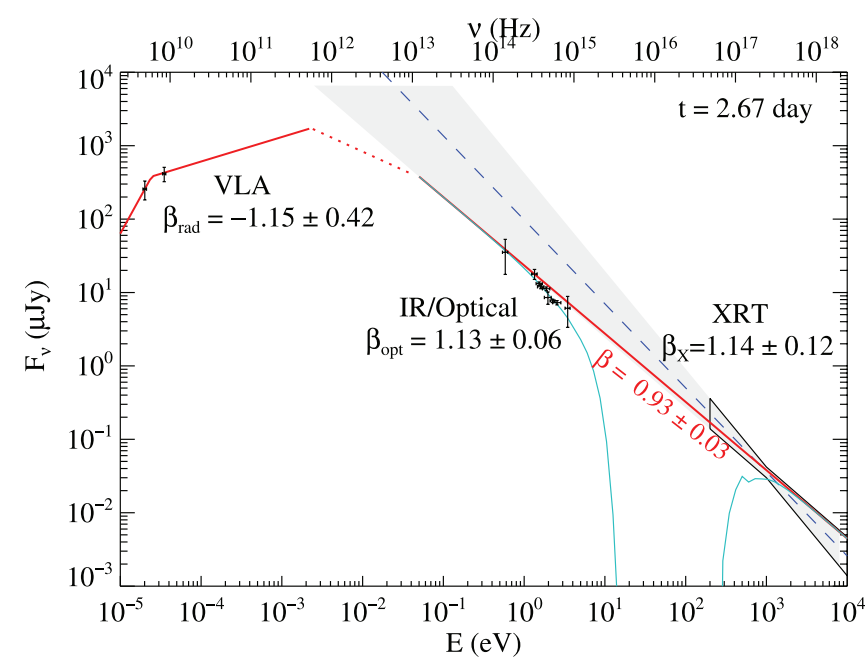

FIG. 14.-Broadband SED at $t=2.67$ days from radio through X-ray observations. The shaded region shows an unbroken extrapolation of the X-ray fit ( $90 \%$ confidence region), which is consistent with the optical measurements. The optical points are corrected for Galactic but not extragalactic extinction; a best-fit model for the effects of host galaxy and intervening galaxy extinction is shown (thin cyan line). The locations of the cooling break and peak frequency shown are arbitrarily chosen; the actual frequencies are not constrained by the available data except that both are located between the radio and optical bands.
TABLE 12

Extinction Models for Optical/X-Ray Fits of GRB 071003

\begin{tabular}{|c|c|c|c|c|}
\hline Model & $A_{V}$ & $R_{V}$ & $\beta$ & $\chi^{2 / \nu}$ \\
\hline None .......................... & 0 & $\ldots$ & $0.913 \pm 0.029$ & $12.4 / 8$ \\
\hline \multicolumn{5}{|c|}{$z=0.372$} \\
\hline Milky Way ............ & $0.239 \pm 0.093$ & 3.09 & $0.939 \pm 0.028$ & $5.80 / 7$ \\
\hline SMC …..................... & $0.209 \pm 0.082$ & 2.74 & $0.934 \pm 0.028$ & $5.95 / 7$ \\
\hline LMC & $0.256 \pm 0.099$ & 3.41 & $0.941 \pm 0.029$ & $5.87 / 7$ \\
\hline Calzetti ...................... & $0.279 \pm 0.108$ & 4.05 & $0.945 \pm 0.029$ & $5.80 / 7$ \\
\hline \multicolumn{5}{|c|}{$z=1.10$} \\
\hline Milky Way ............ & $0.133 \pm 0.058$ & 3.09 & $0.935 \pm 0.029$ & $7.16 / 7$ \\
\hline SMC ….......................... & $0.127 \pm 0.052$ & 2.74 & $0.935 \pm 0.028$ & $6.38 / 7$ \\
\hline LMC .............................. & $0.132 \pm 0.057$ & 3.41 & $0.934 \pm 0.028$ & $7.16 / 7$ \\
\hline Calzetti ...................... & $0.247 \pm 0.095$ & 4.05 & $0.957 \pm 0.032$ & $5.78 / 7$ \\
\hline \multicolumn{5}{|c|}{$z=1.60$} \\
\hline Milky Way ............ & $0.139 \pm 0.048$ & 3.09 & $0.943 \pm 0.028$ & $3.94 / 7$ \\
\hline SMC ….......................... & $0.096 \pm 0.037$ & 2.74 & $0.934 \pm 0.028$ & $5.77 / 7$ \\
\hline LMC .......................... & $0.131 \pm 0.045$ & 3.41 & $0.940 \pm 0.028$ & $3.98 / 7$ \\
\hline Calzetti ...................... & $0.240 \pm 0.093$ & 4.05 & $0.965 \pm 0.033$ & $5.84 / 7$ \\
\hline
\end{tabular}

Notes.-Results of various fits to the contemporaneous optical and X-ray fluxes for extinction due to either the host galaxy or the intervening absorbers at $z=0.372$ and 1.10 . A small amount of extinction is required to accurately fit the data, but its nature is not strongly constrained. We adopt SMC-like extinction at $z=0.372$ in the discussion and plots based on the relative strength of the intervening absorber at this redshift in the spectrum.

constrained to be $0.94 \pm 0.03$, averaged across the different models. This is consistent (although marginally, at about the $90 \%$ confidence level) with the absorption-corrected X-ray measurement of $\beta=1.14 \pm 0.12$.

As expected, the spectrum turns over dramatically somewhere redward of the optical and is declining with decreasing frequency by the radio band. The radio results are discussed further in $\S 5.4$.

\subsection{Photometric Limits on a Host Galaxy and Intervening Absorbers}

Neither our LRIS imaging nor our late-time NGS AO imaging show any evidence of extension or host galaxy emission consistent with the afterglow position. We searched for emission from a host coincident with the OA position by smoothing and binning the PSF-subtracted AO image. No host emission was detected to a conservative upper limit of $K^{\prime} \approx 23$ Vega mag.

In our first-night LRIS image (when the seeing was best and contamination from the bright nearby star relatively minimized), a faint, extended source is visible slightly southwest of the OA. The same source is also visible in the AO image, clearly resolved into a faint galaxy with $K^{\prime} \approx 19$ mag at an offset of $2.07^{\prime \prime}$ southwest of the OA.

We know from the spectral analysis that there are at least four systems that intersect the sight line between the $z=1.604 \mathrm{GRB}$ and Earth, including the host itself. Of these, the strongest candidate for association with the observed galaxy is clearly the $z=0.372$ system, which both is closest and exhibits the strongest absorption signature. (Unfortunately, we have no spectra of the galaxy to confirm this.) This source appears to be a small irregular galaxy, which at this redshift would be offset by $\sim 10 \mathrm{kpc}$ (a reasonable distance to explain the observed absorption) and approximately $0.5 \mathrm{kpc}$ in half-light radius. 
No other extended sources are detected within $3^{\prime \prime}$ of the afterglow, so our upper limit rules out detection of both a host galaxy and any absorbing systems within this distance. The corresponding limit on a galaxy luminosity is only mild, compared to the known GRB host distribution. At the presumptive GRB redshift of $z=1.6$, any host galaxy is limited to a $K$-band absolute magnitude of $M\left(K^{\prime}\right)=-22.2$ Vega mag.

This value falls roughly in the middle of the typical range of previously studied GRB hosts, which appear to have $K$-band luminosities on the order of $0.1 L_{*}$ and are bluer and fainter than typical SCUBA galaxies (Le Floc'h et al. 2003).

\subsection{Spectroscopic Constraints on the Host Galaxy and Intervening Absorbers}

The very weak absorption at the host redshift in our spectrum suggests a lower than average $\mathrm{H}$ i column density along the sight line and/or a metal-poor gas. Because of our low spectral resolution, however, the absorption is unresolved and the line profiles may be saturated (Prochaska 2006). We may conservatively report a lower limit to the column densities by assuming the weak limit. In this manner, we estimate $N_{\mathrm{Mg}^{+}}>10^{12.6} \mathrm{~cm}^{-2}$ based on the equivalent width of $\mathrm{Mg}$ II $\lambda 2803$. For a solar-metallicity gas, this implies $\log N_{\mathrm{H}_{\mathrm{I}}}>10^{17} \mathrm{~cm}^{-2}$. This is a conservative estimate because the gas metallicity is presumably subsolar. Nevertheless, it is unlikely that the gas has an $\mathrm{H}$ i column density matching the values typical of most GRBs.

In addition to the gas associated with GRB 071003, the afterglow spectrum reveals three foreground $\mathrm{Mg}$ II absorbers. Two of these have moderate rest-frame equivalent widths $\left(W_{2796} \approx\right.$ $0.7 \AA$ ), but the lowest redshift system exhibits a very large value $\left(z=0.3722, W_{2796}=2.5 \AA\right)$. The incidence of such strong Mg II absorption at $z<0.5$ has not yet been established along quasar sight lines. These absorbers are very rare at $z \approx 0.5$, however, and the incidence is declining with redshift (Nestor et al. 2005; Prochter et al. 2006a). The number of absorbers with $W_{2796}>1 \AA$ per unit redshift is $\ell(z)=0.13$ at $z=0.5$, and the incidence of absorbers with $W_{2796}>2 \AA$ is an order of magnitude lower. This implies that one would need to observe of order 100 quasar sight lines to detect a single absorber with $W_{2796}>2 \AA$ at $z<0.5$. Although these are a posteriori statistics, this analysis reminds one of the apparent enhancement of strong Mg II absorbers along GRB sight lines (Prochter et al. 2006b). Given its low redshift, this system will be an excellent case to perform follow-up observations and examine the properties of the galaxies hosting such systems (L. K. Pollack et al. 2008, in preparation) The bright nearby star, however, poses a formidable obstacle for non-AO ground-based observations.

\subsection{Energetics}

The measured gamma-ray fluence of $5.32_{-0.67}^{+0.30} \times 10^{-5} \mathrm{ergs} \mathrm{cm}^{-2}$ (Konus, $20 \mathrm{keV}-4 \mathrm{MeV}$; Golenetskii et al. 2007) can be converted to an isotropic-equivalent total energy release in the host frame: $E_{\text {iso }}=3.4_{-0.6}^{+0.2} \times 10^{53} \mathrm{ergs}$, well in the upper range of Swift events.

No clear jet break is observed over the course of our observations, in either the optical bands or the X-ray, out to at least $6 \times$ $10^{5} \mathrm{~s}$. There is a possible monochromatic break in the radio bands at around 8 days $\left(7 \times 10^{5} \mathrm{~s}\right)$, but it appears likely to be a scintillation artifact (see $\S 4.6$ ).

Using this limit, and following Sari et al. (1999) for a uniform circumburst medium, we can calculate the minimum jet opening angle and minimum collimation-corrected energy. Using standard values for the radiative efficiency $(\eta=0.5)$ and circumburst den- sity $\left(n=3.0 \mathrm{~cm}^{-3}\right)$ (the end result is nearly insensitive to these parameters), we have

$$
\begin{aligned}
\theta_{\text {jet }}= & 6.5^{\circ}\left(\frac{t_{\text {jet }}}{\text { days }}\right)^{3 / 8}\left(\frac{n}{3 \mathrm{~cm}^{-3}}\right)^{1 / 8} \\
& \times\left(\frac{1+z}{2}\right)^{-3 / 8}\left(\frac{E_{\text {iso }} / \eta}{10^{53} \mathrm{ergs}}\right)^{-1 / 8} .
\end{aligned}
$$

However, as we discuss later, the late-time afterglow behavior in this case favors a wind model. Thus, following Li \& Chevalier (2003), we have

$$
\theta_{\text {jet }}=5.4^{\circ}\left(\frac{t_{\text {jet }}}{\text { days }}\right)^{1 / 4}\left(A_{*}\right)^{1 / 4}\left(\frac{1+z}{2}\right)^{-1 / 4}\left(\frac{E_{\text {iso }} / \eta}{10^{53} \mathrm{ergs}}\right)^{-1 / 4} .
$$

The upper limit on $t_{\text {jet }}$ of 7 days gives a limit on the opening of at least $3.1\left(A_{*} / 0.1\right)^{1 / 4} \mathrm{deg}$. (As discussed later in $\S 5.4$, we estimate $A_{*} \approx 0.1$ from the broadband spectrum.) Therefore, the collimationcorrected energy is at least $E_{\gamma} \gtrsim 2 \times 10^{50}\left(A_{*} / 0.1\right)^{1 / 2}$ ergs.

It is also possible that the jet break is hidden by the complicated evolution of the burst, including the rebrightening, which would imply more modest energetics for this burst. However, as the late-time slope is still relatively shallow ( $\alpha=1.72$; generally we expect $\alpha \geq 2$ after a jet break), we consider this relatively unlikely.

\section{DISCUSSION}

\subsection{Initial Power-Law Decline}

We first turn our attention to the rapidly declining power law. The temporal behavior of this feature is quite simple, with a decay constant $\alpha=1.466 \pm 0.006$ and no evident substructure before the "bump" or after it. There is no evidence of a rising component or any early break. The observed spectral index $\beta=$ $0.62 \pm 0.33$, although if the extinction measured at late times is also present at early times (as we expect), the intrinsic index is actually shallower; correcting this using our preferred extinction model, we derive $\beta=0.29 \pm 0.49$.

Especially when the decay is observed to flatten later, very early time decay of this nature is often interpreted as a reverse shock. This seems possible: the spectral and temporal indices are within the range of predictions for reverse shock models (specifically, the thick-shell case of Kobayashi 2000). However, a forward shock origin is also consistent. Examining the standard closure relations between $\alpha$ and $\beta$ (as in, for example, Price et al. 2002), all environment models (ISM, wind, and jet) are consistent with the constraints derived from the data, largely because the early-time constraint on $\beta$ is poor. (We discuss the forward vs. reverse shock models for this emission again in $\S 5.3$, in connection with the late-time rebrightening.)

\subsection{The Bump: Internal Shock Origin without a Prompt-Emission Connection}

The bump feature is of considerable interest, since it is nearly simultaneous with a prompt-emission pulse. However, as discussed earlier, the temporal analysis seems to disfavor the interpretation as a prompt reverberation: the bump seems to be already rising even before the prompt spike.

Another possible explanation for the origin of this feature is a large density variation in the surrounding medium (a large clump 
or other discrete physical feature in the path of the expanding shock). The observed pulse width $\Delta t / t \approx 1$ is consistent with a density variation, and the general appearance of the light curve over this region is reminiscent of simulations of a GRB forward shock intersecting ISM density enhancements (e.g., Fig. 3 of Nakar \& Piran 2003). However, our observation of possible color change across the bump would (if real) disfavor this hypothesis, at least in the simplest models: density variation will not change the intrinsic spectrum, unless either the microphysical parameters or cooling frequency suddenly and significantly change. We consider this unlikely, although some authors (e.g., Yost et al. 2003; Granot \& Kumar 2006) have discussed the role of variable microphysics in previous GRB afterglows.

Alternatively, the observation that the fast-declining component seems completely unaffected by the afterglow (the temporal indices before and after are effectively identical) leads us to interpret the bump as originating from a distinct emission episode: given the rapid rise and fall and the hint of blue-to-red evolution, we suggest that it arises from internal-shock emission. Hard-tosoft evolution and an underlying power-law decay not affected by the flare have also been seen in X-ray flares (Butler \& Kocevski 2007a; Chincarini et al. 2007). We also note that earlier studies of GRB prompt emission have shown pulses observed at lower energy to be broader than those at higher energy (Fenimore et al. 1995); this trend may continue into the optical band. The broader, smoother profile of this pulse relative to the much faster evolving $\mathrm{X}$-ray flares may in this case illustrate important attributes of the emission, either from viewing effects or resulting from the physics of the emission itself.

\subsection{The Late Rebrightening}

The rebrightening phase of this burst is quite dramatic. While our observations do not sample the peak of the emission, a fit with a reasonable assumption of the sharpness parameter suggests that the flux increased by approximately $1 \mathrm{mag}$, and the amount of integrated optical flux released during the rebrightening is comparable to or more than that emitted by the early afterglow. A rise in optical flux of more than a magnitude at intermediate times (well after the end of prompt emission, but before any supernova component) has to our knowledge been seen in only a handful of previous cases: GRB 970508 (Castro-Tirado et al. 1998), GRB 041219A (Blake et al. 2005), GRB 060729 (Grupe et al. 2007), GRB 070420 (Jelínek et al. 2007), and GRB 070311 (Guidorzi et al. 2007).

The rebrightening is also notable because it appears to differ subtly from the early decay, even though the evolution of both curves is generally quite simple. The decay index and spectral index both steepen, by $\Delta \alpha=0.25 \pm 0.14$ and $\Delta \beta=0.80 \pm 0.30$, respectively. Assuming a synchrotron spectrum, there are only two possible origins for this: the optical band is in different synchrotron regimes at different times (specifically, $\nu<\nu_{c}$ before cooling, and $\nu>\nu_{c}$ after cooling, consistent with the changes observed), or because of a shift in the electron index $p$ by approximately $\Delta p=+0.4$.

We consider several physical origins for the rebrightening feature: the appearance of the forward shock when the burst ejecta first decelerate against the ISM, the late-time peak of a preexisting forward shock due to evolution of the critical frequencies, impact of the forward shock through a density variation, and rebrightening caused by a refreshed shock.

Appearance of forward shock.-When the GRB ejecta first begin to sweep up an amount of matter from the ISM comparable to the energy in the ejecta, they begin to decelerate, and reverse and forward shocks are propagated back into the ejecta and forward into the ISM, respectively; depending on the Lorentz factor, both shocks can then rise very quickly. We consider this scenario extremely unlikely to be relevant, since by necessity the forward and reverse shocks must rise simultaneously, and there is no explanation for the bright early-time component in the burst, save for a prompt model connected with internal shocks, but as we have already shown, there is no evidence linking the early optical behavior with the high-energy emission.

Spectral peak of existing forward shock.-A more reasonable model postulates that the reverse and forward shocks both formed extremely early, but because they evolve differently (the reverse shock, whose synchrotron parameters are boosted down by factors of $\gamma^{2}$, begins to fade immediately, while the forward shock will rise at lower frequencies), the reverse shock fades rapidly, while the forward shock can rise and peak when the synchrotron frequency $\nu_{m}$ passes through the optical band. This model has, for example, been invoked to explain early-time bumps in the light curves of GRB 021004 (e.g., Kobayashi \& Zhang 2003), GRB 050525A (Shao \& Dai 2005), and GRB 080319B (Bloom et al. 2008), which level off significantly (but do not rebrighten) at around $10^{4} \mathrm{~s}$. However, this model is problematic here: although we have only sparse observations of the rebrightening, the observed rising temporal index of $\alpha=-1.12 \pm 0.16$ is far too fast to be consistent with a rising phase of a forward adiabatic shock, which predicts $F \propto t^{(2-s) /(4-s)}\left(=t^{1 / 2}\right.$ for a constant-density ISM and constant for a wind). Therefore, the synchrotron peak of the forward shock alone cannot explain this feature.

Density variation.-A third possibility, not invoking the transition between reverse and forward shocks, might be a dramatic density variation: for example, the impact of the shock wave into a previously ejected circumstellar shell, or emergence of the shock from a low-density cavity into a dense external medium. Density fluctuations have been successfully invoked to explain low-level variations in several previous studies (e.g., Lazzati et al. $2002)$, and the timescale of the rebrightening $(\Delta t / t \approx 1)$ is consistent with a density fluctuation origin (Nakar \& Piran 2003). However, in this case we would expect neither a change in the spectral index (as is probably observed) nor such a slow decline after the peak, with a temporal index that differs significantly but only slightly from the value of the initial decay. Furthermore, detailed numerical studies by numerous authors (Huang et al. 2006; Nakar \& Piran 2003; Nakar \& Granot 2007) have failed to reproduce anything but the smallest rebrightening signatures in previous GRBs using density variations.

Multicomponent jet.- - The complicated light curve of GRB 030329 has been interpreted (Berger et al. 2003) as the result of two separate forward shocks, arising from two different jet components: a narrow, highly relativistic jet whose emission peaks extremely early, plus a wide, more mildly relativistic jet that dominates the late-time and radio evolution. Could this model conceivably explain the observations of GRB 071003 ? While a complete analysis is beyond the scope of this paper, we note that the observations do seem consistent: the similarities of late-time decay of both rapid and late-time components are naturally explained, the timescale of our rebrightening is similar to that observed in GRB 030329, and (notably) the most significant criticism of the two-jet interpretation of GRB 030329 (that the rebrightening rose too rapidly and peaked too sharply; Huang et al. 2006) does not apply here: the rebrightening in this case is much smoother than that observed for GRB 030329.

Refreshed shock.-Finally, we consider the possibility that this feature is due to a discrete energy reinjection energizing the forward shock, such as via a slow-moving shell that catches up to the forward shock at late times after it decelerates. This seems 
consistent with all observations, although largely by virtue of not making strong predictions; by invoking a customized pattern of energy reinjection at the right times, a very broad space of light-curve behavior can be modeled (Huang et al. 2006). We do note that a large, sudden rebrightening of this nature may also produce a (second) reverse shock, which would be observable in radio and decline rapidly with time. The radio flux does in fact decay somewhat (in contrast to the expectation from a forward shock model, where the radio flux is constant or rising), and the measured $\alpha=0.33 \pm 0.10$ is not far from the predicted decay constant for a reverse shock of $\alpha \approx \frac{1}{2}$ in the $\nu<\nu_{m}$ frequency regime (Kobayashi 2000). However, the radio decay could conceivably be due to other effects (e.g., late jet break), and without an independent measurement of the synchrotron peak frequency $\nu_{m}$ and late-time Lorentz factor $\Gamma$ we are unable to further constrain the presence or absence of such a feature with the limited observations available.

We therefore find that only the multicomponent jet and refreshed shock models are consistent with all available data. Unfortunately, we do not have sufficient observations during the rising phase of the rebrightening to distinguish the two models; in particular, we can set no constraints on the color evolution and lack a detailed light curve of the rise to peak of the rebrightening. We do note that the X-ray observations are already decaying well before the (probable) optical peak by an extrapolation of our observations (Fig. 10), which may suggest hard-to-soft evolution in this feature as well. However, as noted earlier, the X-ray decay extrapolates back to the BAT light curve without explicit need for a rebrightening, so without earlier X-ray measurements this association is speculative.

\subsection{Environmental Constraints}

In the simplest models, the late-time light curve of any GRB is fixed by a number of basic parameters: microphysical parameters $\epsilon_{B}$ (the fraction of energy in magnetic fields), $\epsilon_{e}$ (the fraction of energy in electrons), and $p$ (the electron energy index); macroscopic parameters $E_{K}$ (the blast wave energy) and $\theta_{j}$ (the jet opening angle); and a parameter quantifying the density of the surrounding medium, $n$ (for a uniform density) or $A_{*}$ (for an $r^{-2}$ density profile). Our broadband observations (spanning from radio to X-rays) should, in principle, allow us to firmly constrain most of these parameters for GRB 071003, or, more accurately, to its late rebrightening phase, as this component is dominant at late times.

The indices $\alpha$ and $\beta$ are both well constrained at late times in the optical through X-ray bands, thanks to the wide range of temporal and spatial sampling: $\alpha_{O+\mathrm{X}}=1.71 \pm 0.14, \beta_{\mathrm{OX}}=0.93 \pm$ 0.03 . Two environment models satisfy these constraints within $90 \%$ confidence: a wind-driven medium $\left(\rho \propto r^{-2}\right)$ in which $p \approx 2.9$, and a model in which the jet break has already occurred with $p \approx 1.9$ (but consistent with $p=2$ ). Notably, ISM models are a poor fit: the late-time decay rate is too fast for the shallow spectral index. The radio observations appear to support this conclusion: the rising light curve predicted by the ISM model is clearly ruled out, and while the slow radio decay $\left(\alpha_{R}=0.33 \pm 0.1\right)$ is inconsistent in detail with the wind prediction of constant evolution as well, it is conceivable that variations from an exact $s=-2$ profile, an additional source of radio emission at early times (e.g., a reverse shock), or a soft jet break at $t \approx 5$ days may explain this difficulty.

The apparent spectral index of $\beta \approx-1.1$ observed in the radio is notable. A synchrotron spectrum is expected to have a selfabsorbed $\beta=-2$ spectrum below the self-absorption frequency $\nu_{a}$ and a spectrum of $\beta=-0.5$ above it. The fact that the observed spectral index is intermediate between these values and consistent with neither (to $~ 90 \%$ confidence) tells us that, if the spectrum is really synchrotron, the absorption break is likely to be very close to these frequencies, although exact constraints are difficult with only two frequencies since the break is likely to be quite soft. The radio evolution appears nearly achromatic, which would argue against this interpretation, but considering the relatively narrow time and frequency window of the observations and unknown break sharpness, we feel that this is not a major concern.

Because the ISM model is notably discrepant with the measured values of $\alpha$ and $\beta$, we unfortunately cannot use the afterglow as a probe of the ambient density. If the wind model, which is more consistent with the observations in this case, is correct, we can calculate the parameter $A_{*}$ using (for example) equation (2) in Chevalier \& Li (1999):

$$
F_{\nu_{m}}=20 \mathrm{mJy}\left(\frac{d_{L}}{5403 \mathrm{Mpc}}\right)(1+z)^{1 / 2}\left(\frac{\epsilon_{B}}{0.1}\right)^{-1 / 2} E_{52}^{1 / 2} A_{*} t_{d}^{1 / 2} .
$$

While we have no direct measurement of $F_{\nu_{m}}$, it is constrained by the radio and optical observations (see Fig. 14) to be $\sim 1 \mathrm{mJy}$ (within a factor of $\sim 3)$. We therefore measure $A_{*}=0.07\left(\epsilon_{B} / 0.1\right)^{1 / 2}$, an interestingly low value regardless of the value of $\epsilon_{B}$. While $\epsilon_{B}$ is not strongly constrained, the absence of a cooling break between the X-ray and optical bands during the first 5 days (the cooling frequency $\nu_{c}$ increases in a wind model) requires $\epsilon_{B} \gtrsim 0.3$.

It is possible that the wind model is inappropriate and the rapid optical decay is due to a jet that broke before our multicolor latetime observations. (One possible criticism of the wind model is that in this case, the color transition between early and late times is hard to explain; because the cooling frequency rises with time, if $\nu>\nu_{c}$ late it must have been early as well under standard synchrotron evolution. However, because the rebrightening appears to be either a separate phenomenon or a large energy impulse that could conceivably have "reset" the synchrotron parameters [including $\nu_{c}$ ] to new values, this may not be a major concern.) No jet break is observed in the light curve, but it is possible that a jet signature was concealed by the rebrightening. This case would certainly rule out the wide-angle jet interpretation of the secondary peak and would significantly reduce the energetics.

\subsection{Spectral Implications on the Environment and Host Galaxy}

The late-time spectroscopy and imaging tell a coherent story: unlike the vast majority of GRBs (Wainwright et al. 2007; Prochaska et al. 2008), GRB 071003 did not occur in a gas-rich ${ }^{28}$ galaxy. The environment is more consistent with a progenitor located in an outer galactic halo, or in an extremely small (even compared to "normal" long-duration GRB hosts) and gas-poor galaxy. While the possibility of line saturation prevents us from setting definitive upper limits, the column density through any host is consistent with being 3 orders of magnitude below typical GRB-derived values, and the contrast to the overall GRB population, which is dominated

\footnotetext{
28 Since our measurement is based on magnesium, we are directly measuring the metal column, not the gas column. An alternate possibility, therefore, is that the host is "normal" but extraordinarily metal-poor, less than $10^{-2}$ of the average solar abundance. However, we consider a highly subluminous host a more likely possibility. Both effects may be in play: low-luminosity galaxies, and those with low equivalent widths, tend to be relatively metal-poor (Prochaska et al. 2008).
} 
by subluminous galaxies to begin with (e.g., Fruchter et al. 2006; Fynbo et al. 2008), is dramatic.

While it is well established that long-duration GRBs generally originate from massive stars, we should be careful to ensure that our prior experience does not blind us to the existence of rarer subclasses of events. We note that one other GRB on record, GRB 070125 , had very similar properties: extremely low Mg II absorption and no coincident host (Cenko et al. 2008), as well as a very bright afterglow and extreme energetics $\left(E_{\gamma}=3 \times 10^{52}\right.$ ergs; Chandra et al. 2008), and even a (mild) late-time rebrightening (Updike et al. 2008). Both are also among the few Swift bursts detected at radio wavelengths.

However, GRB 070125 and GRB 071003 show evidence from their broadband light curves of origins typical of ordinary long GRBs. In the case of GRB 070125, a constant but very high circumstellar density suggested that it occurred in what was locally a dense environment, not an empty galactic halo, despite the near absence of a large-scale gas signature in the spectrum. In our case, for GRB 071003, we find evidence of a windlike stratified environment, a characteristic of a massive star. Together, these events appear to suggest an origin for these "halo" bursts similar to those of all other GRBs.

If GRB 071003 did occur in a star-forming region, then there are two possibilities consistent with the extremely small metal absorption in the spectrum. First, the burst may simply have formed in an extremely subluminous galaxy: necessarily, the number or distribution of such objects at very high redshift is not observationally constrained, but most simulations predict an abundance of small, highly sub-Galactic halos in the universe that could very well harbor limited star formation. Alternatively, GRB 071003 may have occurred in a tidally stripped tail from another, larger galaxy. In this case, further follow-up observations should reveal a disturbed, star-forming host in the close vicinity of the burst.

Either scenario seems plausible to explain the constraints derived on the burst environment. In either case, if GRBs are shown to be reasonable tracers of star formation at high redshift, then future large-sample GRB spectroscopy missions may be able to place important constraints on the star formation history of the universe not possible by any other means. While the sample size of such low column density GRBs is now small (two events, with GRB 061021 [Thoene et al. 2006] possibly constituting a third example), these results are already suggestive that this fraction may be significant (on the order of a few percent), and systematic rapid afterglow spectroscopy should continue to increase the number considerably over the years and decades to come. It would be an interesting discovery if the distribution of $\mathrm{Mg}$ II equivalent widths turns out to be bimodal.

On a related note, the existence of GRB 071003 and GRB 070125 may have important implications regarding the escape fraction of ionizing photons and the reionization history of the universe. Although the relatively low redshift of these systems keeps the Ly $\alpha$ and Lyman break absorption features out of our spectral range and prevents us from measuring the $\mathrm{H}$ i column density directly (Chen et al. 2007), these GRBs provide evidence that massive stars can form well outside of gas-dense hosts, where there is little to shield the intergalactic medium from their ionizing UV radiation. If the fraction of these events is more than a few percent at $z>7$, then such "halo" stars may in fact be primarily responsible for the reionization of the universe. Observationally, spectroscopy of such events at these high redshifts may allow accurate measurement of the neutral gas fraction $\bar{x}_{\mathrm{H}}$ (e.g., McQuinn et al. 2008) without the interference of saturated line profiles originating from the host galaxy.

\section{CONCLUSIONS}

Although the temporal evolution of the optical afterglow of GRB 071003 is complicated, our early- through late-time photometric follow-up data clearly resolve the optical light curve into separate components. Observations from KAIT during the prompt phase of the GRB revealed a slowly rising, slowly falling bump or flare component, superimposed on a simple fading power law that has no observable correlation with the prompt emission, suggesting that while early internal-shock flares can be observed in the optical, they are not necessarily the same as those producing the highenergy signatures. Our late-time observations revealed one of the most dramatic late rebrightenings ever recorded in a GRB light curve and suggest that this feature is not due to a reverse-forward shock transition or density variation, requiring either angular jet structure or very discrete late-time reenergizing of the optical afterglow. This may have important implications for the interpretation of other, less dramatic bumps and rebrightenings at similar timescales that appear to be common features in GRB afterglows.

The spectroscopic study of GRB 071003 offers a cautionary tale about the standard use of $\mathrm{Mg}$ II to infer a redshift: while it is common practice to use the highest redshift $\mathrm{Mg}$ II system observed (especially in the cases when the absorption is quite strong) under the assumption that the GRB host system should show significant metal absorption, here we have a clear case where this assumption is fundamentally flawed. Were the $\mathrm{S} / \mathrm{N}$ of the spectrum worse, or the host galaxy absorption even weaker by a factor of only $2-3$, it is likely that we would have missed the higher redshift system entirely and proceeded with the assumption that this burst was at a redshift of 1.100 instead of 1.604. In light of this fact, previous and future GRB redshift claims based solely on identification of $\mathrm{Mg}$ II absorption should be regarded with increased skepticism.

The intervening absorption systems are nevertheless also remarkable. With three completely independent $\mathrm{Mg}$ II systems along the line of sight, GRB 071003 is among the most dramatic examples yet of the bizarre overabundance of these systems in GRB afterglows relative to those of quasars. Further study of this sight line, especially using AO systems, may help shed light on this mysterious result.

KAIT and its ongoing operation were made possible by donations from Sun Microsystems, Inc., the Hewlett-Packard Company, AutoScope Corporation, Lick Observatory, the National Science Foundation, the University of California, the Sylvia \& Jim Katzman Foundation, and the TABASGO Foundation. J. S. B.'s group is supported in part by the Hellman Faculty Fund, Las Cumbres Observatory Global Telescope Network, and NASA/ Swift Guest Investigator grant NNG05GF55G. A. V. F.'s group is supported by NSF grant AST 06-07485 and the TABASGO Foundation, as well as by NASA/Swift Guest Investigator grants NNG05GF35G and NNG06GI86G. N. R. B. is partially supported by a SciDAC grant from the Department of Energy. J. X. P. is partially supported by NASA/Swift Guest Investigator grant NNG05GF55G and NSF CAREER grant AST 05-48180. H. Swan has been supported by NSF grant AST 03-35588 and by the Michigan Space Grant Consortium. F. Yuan has been supported under NASA/Swift Guest Investigator Grant NNX-07AF02G. We acknowledge helpful discussions with E. Ramirez-Ruiz and thank D. Whalen and A. Heger for their calculations of the photon flux from massive stars. We offer particular thanks to D. A. Kann for useful discussions and feedback.

This research is based in part on observations obtained at the Gemini Observatory, which is operated by the Association of 
Universities for Research in Astronomy, Inc., under a cooperative agreement with the NSF on behalf of the Gemini partnership. Some of the data presented herein were obtained at the W. M. Keck Observatory, which is operated as a scientific partnership among the California Institute of Technology, the University of California, and NASA; the Observatory was made possible by the generous financial support of the W. M. Keck Foundation. We wish to extend special thanks to those of Hawaiian ancestry on whose sacred mountain we are privileged to be guests. We are grateful to the staffs at the Gemini, Keck, and Lick Observatories for their assistance.
Akerlof, C., et al. 1999, Nature, 398, 400

Alard, C., \& Lupton, R. H. 1998, ApJ, 503, 325

Barris, B. J., Tonry, J. L., Novicki, M. C., \& Wood-Vasey, W. M. 2005, AJ, 130,2272

Berger, E., et al. 2003, Nature, 426, 154

Bertin, E., \& Arnouts, S. 1996, A\&AS, 117, 393

Beuermann, K., et al. 1999, A\&A, 352, L26

Blake, C. H., et al. 2005, Nature, 435, 181

Bloom, J. S., Kulkarni, S. R., \& Djorgovski, S. G. 2002, AJ, 123, 1111

Bloom, J. S., et al. 2008, ApJ, submitted (arXiv:0803.3215)

Butler, N. R., \& Kocevski, D. 2007a, ApJ, 663, 407 2007b, ApJ, 668, 400

Butler, N. R., et al. 2006, ApJ, 652, 1390

Calzetti, D., Armus, L., Bohlin, R. C., Kinney, A. L., Koornneef, J., \& StorchiBergmann, T. 2000, ApJ, 533, 682

Castro-Tirado, A. J., et al. 1998, Science, 279, 1011

Cenko, S. B., \& Fox, D. B. 2007, GCN Circ. 6839, http:/gen.gsfe.nasa.gov/ $\mathrm{gcn} / \mathrm{gcn} 3 / 6839 . \mathrm{gcn} 3$

Cenko, S. B., et al. 2006, PASP, 118, 1396 2008, ApJ, 677, 441

Chandra, P., et al. 2008, ApJ, 683, 924

Chen, H.-W., Prochaska, J. X., \& Gnedin, N. Y. 2007, ApJ, 667, L125

Chevalier, R. A., \& Li, Z.-Y. 1999, ApJ, 520, L29

Chincarini, G., et al. 2007, ApJ, 671, 1903

D'Elia, V., et al. 2007, A\&A, 467, 629 . 2008, ApJ, submitted (arXiv:0804.2141)

Dessauges-Zavadsky, M., Chen, H.-W., Prochaska, J. X., Bloom, J. S., \& Barth, A. J. 2006, ApJ, 648, L89

Dickey, J. M., \& Lockman, F. J. 1990, ARA\&A, 28, 215

Fenimore, E. E., in 't Zand, J. J. M., Norris, J. P., Bonnell, J. T., \& Nemiroff, R. J. 1995, ApJ, 448, L101

Filippenko, A. V. 1982, PASP, 94, 715

- 2005, in ASP Conf. Ser. 332, The Fate of the Most Massive Stars, ed. R. Humphreys \& K. Stanek (San Francisco: ASP), 33

Filippenko, A. V., Li, W. D., Treffers, R. R., \& Modjaz, M. 2001, in IAU Colloq. 183, Small Telescope Astronomy on Global Scales, ed. B. Paczyński, W.-P. Chen, \& C. Lemme (ASP Conf. Ser. 246; San Francisco: ASP), 121

Fitzpatrick, E. L., \& Massa, D. 1990, ApJS, 72, 163

Flewelling-Swan, H., et al. 2006, BAAS, 38, 134

Fruchter, A. S., et al. 2006, Nature, 441, 463

Fugazza, D., et al. 2007, GCN Circ. 6851, http://gcn.gsfc.nasa.gov/gcn/gcn3/ 6851.gen3

Fynbo, J. P. U., Prochaska, J. X., Sommer-Larsen, J., Dessauges-Zavadsky, M., \& Moller, P. 2008, ApJ, 683, 321

Garnavich, P. M., Loeb, A., \& Stanek, K. Z. 2000, ApJ, 544, L11

Gehrels, N. 2007, GCN Circ. 6760, http://gcn.gsfc.nasa.gov/gen/gcn3/6760 gen 3

Gehrels, N., et al. 2004, ApJ, 611, 1005

Golenetskii, S., Aptekar, R., Mazets, E., Pal'shin, V., Frederiks, D., \& Cline, T. 2007, GCN Circ. 6849, http://gcn.gsfc.nasa.gov/gcn/gcn3/6849.gcn3

Granot, J., \& Kumar, P. 2006, MNRAS, 366, L13

Grupe, D., et al. 2007, ApJ, 662, 443

Guidorzi, C., et al. 2007, A\&A, 474, 793

Holland, S. T., et al. 2003, AJ, 125, 2291

Huang, Y. F., Cheng, K. S., \& Gao, T. T. 2006, ApJ, 637, 873

Jelínek, M., Tristram, P., Postigo, A. d. U., Gorosabel, J., Hale, F., Gilmore, A., Kilmartin, P., \& Castro-Tirado, A. J. 2007, GCN Circ. 6338, http://gcn.gsfc .nasa.gov/gcn $/ \mathrm{gcn} 3 / 6338 . \mathrm{gcn} 3$

Jester, S., et al. 2005, AJ, 130, 873

Kobayashi, S. 2000, ApJ, 545, 807

Kobayashi, S., \& Zhang, B. 2003, ApJ, 582, L75

Kocevski, D., Butler, N., \& Bloom, J. S. 2007, ApJ, 667, 1024

Landolt, A. U. 1992, AJ, 104, 340

Laursen, L. T., \& Stanek, K. Z. 2003, ApJ, 597, L107

Lazzati, D., Rossi, E., Covino, S., Ghisellini, G., \& Malesani, D. 2002, A\&A, 396, L5

Le Floc'h, E., et al. 2003, A\&A, 400, 499

Li, W. 2006, GCN Circ. 4727, http://gcn.gsfc.nasa.gov/gcn/gen3/4727.gen3 . 2007, GCN Circ. 6838, http://gen.gsfc.nasa.gov/gen/gen3/6838.gen3

\section{EFERENCES}

Li, W., Chornock, R., Filippenko, A. V., Perley, D. A., \& Bloom, J. S. 2007, GCN Circ. 6844, http://gen.gsfc.nasa.gov/gen/gen3/6844.gen3

Li, W., Filippenko, A. V., Chornock, R., \& Jha, S. 2003a, ApJ, 586, L9 2003b, PASP, 115, 844

Li, W. D., et al. 2000, in AIP Conf. Proc. 522, Cosmic Explosions: Tenth Astrophysics Conference, ed. S. S. Holt \& W. W. Zhang (New York: AIP), 103

Li, Z.-Y., \& Chevalier, R. A. 2003, in Supernovae and Gamma-Ray Bursters, ed. K. Weiler (Berlin: Springer), 419

McQuinn, M., Lidz, A., Zaldarriaga, M., Hernquist, L., \& Dutta, S. 2008, MNRAS, 388, 1101

Misra, K., Pandey, S. B., Roy, R., \& Castro-Tirado, A. J. 2007, GCN Circ. 6840, http://gen.gsfc.nasa.gov/gen/gen3/6840.gen3

Monet, D. G., et al. 2003, AJ, 125, 984

Nakar, E., \& Granot, J. 2007, MNRAS, 380, 1744

Nakar, E., \& Piran, T. 2003, ApJ, 598, 400

Nestor, D. B., Turnshek, D. A., \& Rao, S. M. 2005, ApJ, 628, 637

Nousek, J. A., et al. 2006, ApJ, 642, 389

Nysewander, M., Reichart, D. E., Crain, J. A., Foster, A., Haislip, J., Ivarsen, K., Lacluyze, A., \& Trotter, A. 2007, ApJ, submitted (arXiv:0708.3444)

Oke, J. B., et al. 1995, PASP, 107, 375

Perley, D. A., Chornock, R., Bloom, J. S., Fassnacht, C., \& Auger, M. W. 2007a, GCN Circ. 6850, http://gcn.gsfc.nasa.gov/gcn/gen3/6850.gcn3

Perley, D. A., Prochaska, J. X., Bloom, J. S., \& Smith, G. 2007b, GCN Circ. 6843, http://gcn.gsfc.nasa.gov/gen/gen3/6843.gcn3

Perley, D. A., et al. 2008, ApJ, 672, 449

Phillips, A. C., Miller, J., Cowley, D., \& Wallace, V. 2006, Proc. SPIE, 6269, 56

Pollack, L. K., Dam, M. A. v., Mignant, D. L., Johansson, E. M., Perley, D. A., \&

Bloom, J. S. 2007, GCN Circ. 7010, http://gcn.gsfc.nasa.gov/gen/gcn3/7010.gcn3 Price, P. A., et al. 2002, ApJ, 572, L51

Prochaska, J. X. 2006, ApJ, 650, 272

Prochaska, J. X., Chen, H.-W., \& Bloom, J. S. 2006, ApJ, 648, 95

Prochaska, J. X., Chen, H.-W., Dessauges-Zavadsky, M., \& Bloom, J. S. 2007, ApJ, 666, 267

Prochaska, J. X., Chen, H.-W., Wolfe, A. M., Dessauges-Zavadsky, M., \& Bloom, J. S. 2008, ApJ, 672, 59

Prochter, G. E., Prochaska, J. X., \& Burles, S. M. 2006a, ApJ, 639, 766

Prochter, G. E., et al. 2006b, ApJ, 648, L93

Ricker, G. R., et al. 2003, in AIP Conf. Proc. 662, Gamma-Ray Burst and Afterglow Astronomy 2001: A Workshop Celebrating the First Year of the HETE Mission, ed. G. R. Ricker \& R. K. Vanderspek (New York: AIP), 3

Rodgers, C. T., Canterna, R., Smith, J. A., Pierce, M. J., \& Tucker, D. L. 2006, AJ, 132, 989

Rykoff, E. S., et al. 2006, ApJ, 638, L5

Sari, R., Piran, T., \& Halpern, J. P. 1999, ApJ, 519, L17

Savaglio, S., Fall, S. M., \& Fiore, F. 2003, ApJ, 585, 638

Schady, P., et al. 2007, GCN Circ. 6837, http://gcn.gsfc.nasa.gov/gcn/gcn3/6837.gcn3

Schmidt, B. P., et al. 1998, ApJ, 507, 46

Shao, L., \& Dai, Z. G. 2005, ApJ, 633, 1027

Shih, C. Y., Lee, Y. H., Urata, Y., \& Huang, K. Y. 2007, GCN Circ. 6846, http:// gen.gsfc.nasa.gov/gen/gen $3 / 6846 . g c n 3$

Stone, R. P. S. 1977, ApJ, 218, 767

Swan, H., Smith, I., Rujopakarn, W., Yuan, F., Yost, S., Akerlof, C., Skinner, M., \& Russell, R. 2007, GCN Circ. 6841, http://gcn.gsfc.nasa.gov/gcn/gcn3/6841.gcn3

Thoene, C. C., Fynbo, J. P. U., \& Jakobsson, P. 2006, GCN Circ. 5747, http:// gen.gsfc.nasa.gov/gen/gen $3 / 5747 . g c n 3$

Updike, A. C., et al. 2008, ApJ, 685, 361

van Dam, M. A., Le Mignant, D., \& Macintosh, B. A. 2004, Appl. Optics, 43, 5458

Vestrand, W. T., et al. 2005, Nature, 435, 178 2006, Nature, 442, 172

Vreeswijk, P. M., et al. 2007, A\&A, 468, 83

Wainwright, C., Berger, E., \& Penprase, B. E. 2007, ApJ, 657, 367

Walker, M. A. 1998, MNRAS, 294, 307 2001, MNRAS, 321, 176

Woźniak, P. R., Vestrand, W. T., Wren, J. A., White, R. R., Evans, S. M., \& Casperson, D. 2006, ApJ, 642, L99

Yost, S. A., Harrison, F. A., Sari, R., \& Frail, D. A. 2003, ApJ, 597, 459

Yost, S. A., et al. 2007, ApJ, 657, 925

Zhang, B., Fan, Y. Z., Dyks, J., Kobayashi, S., Mészáros, P., Burrows, D. N., Nousek, J. A., \& Gehrels, N. 2006, ApJ, 642, 354 